\title{
Asymptotic laws for tagged-particle motion in glassy systems
}

\author{
M. Fuchs, W. Götze, and M. R. Mayr \\ Physik Department, Technische Universität München, 85747 Garching, Germany
}

(Received 31 March 1998)

\begin{abstract}
Within mode-coupling theory for structural relaxation in simple systems, the asymptotic laws and their leading-asymptotic correction formulas are derived for the motion of a tagged particle near a glass-transition singularity. These analytic results are compared with numerical ones of the equations of motion evaluated for a tagged hard sphere moving in a hard-sphere system. It is found that the long-time part of the two-step relaxation process for the mean-squared displacement can be characterized by the $\alpha$-relaxation scaling law and von Schweidler's power-law decay, while the critical-decay regime is dominated by the corrections to the leading power-law behavior. For parameters of interest for the interpretations of experimental data, the corrections to the leading asymptotic laws for the non-Gaussian parameter are found to be so large that the leading asymptotic results are altered qualitatively by the corrections. Results for the non-Gaussian parameter are shown to follow qualitatively the findings reported in the molecular-dynamics-simulations work by Kob and Andersen [Phys. Rev. E 51, 4626 (1995)]. [S1063-651X(98)09609-3]
\end{abstract}

PACS number(s): 64.70.Pf, 61.20.Lc

\section{INTRODUCTION}

Mode-coupling theory (MCT) for the structural relaxation of glassy liquids $[1,2]$ has provided explanations for longknown phenomena, such as, for example, the stretching of the $\alpha$ process, the time-temperature superposition principle, and the von Schweidler law. It has also predicted new phenomena such as a square-root singularity in the temperature dependence of the Debye-Waller factor and the critical decay. For details, the reader is referred to Refs. [3,4] and the papers quoted there. The universal features of the MCT arise as leading-order results near a bifurcation singularity, which was identified as an ideal glass transition-in reality smeared out due to additional relaxation processes. These theoretical findings also provided some motivation for a number of experiments focusing on the evolution of structural relaxation in the vicinity of the predicted glass-transition singularity (see Refs. [5-17] and the papers quoted there). In turn, these experiments sparked off new theoretical developments such as the derivation and discussion of next-to-leading-order asymptotic results in the vicinity of the bifurcation singularity [18], and the extension of the theory to liquids consisting of or containing nonspherical molecules $[19,20]$. Using the coherent intermediate scattering function of a hard-sphere system (HSS) as an example, the preceding work [18] illustrates how asymptotic corrections explain the deviation from the leading-order results, and shows how general properties of these corrections may be included into a quantitative analysis of experimental data. Due to the presence of additional amplitudes and a new second-order scaling function, many features of these corrections are different for different observables, used for the description of the glassy dynamics. Therefore, we intend to extend the previous work to a discussion of the conceptually simplest functions characterizing the dynamics, namely, the density correlator of a tagged particle and its special limits, the mean-squared displacement, and the non-Gaussian parameter.

The tagged-particle density correlators can be measured by a variety of techniques. Neutron scattering from molecu- lar liquids with an incoherent scattering cross section such as orthoterphenyl $[17,21]$ probes its spectrum. For a glassy colloidal suspension the correlator has recently been measured by dynamic light scattering [22]. From measurements of the incoherent intermediate scattering function for small wave vectors, one can extract the mean-squared displacement [2224] and, in principle, also information about the nonGaussian parameter. In computer simulations the taggedparticle correlator is a preferred quantity-compared to the coherent density fluctuation function-due to statistical advantages. The mean-squared displacement and the nonGaussian parameter can also be directly extracted from computer simulation data $[11,25]$. The reported simulation results for the non-Gaussian parameter challenged the universal features predicted by MCT. Within the accessible simulationtime window, the non-Gaussian parameter showed no signs of the predictions of a two-step relaxation process nor of the time-temperature superposition principle, even though other dynamical quantities such as the intermediate scattering function, self-intermediate scattering function, and meansquared displacement fitted into the picture for glassy dynamics drawn by MCT.

To proceed, equations of motion will be derived for the mean-squared displacement and the non-Gaussian parameter. The relaxation kernels in those equations are given by modecoupling functionals, which require correlators of the density and the tagged-particle density as input. For the latter, previously derived MCT equations [2] are used. Then an asymptotic expansion of the solutions will be carried out. All equations will be solved and studied in quantitative detail for the model system of a tagged hard sphere immersed in a HSS. The HSS has already been the subject of a number of theoretical investigations, as can be inferred from Refs. $[18,26]$ and the papers quoted there. Its importance stems in part from the fact that it is the simplest system for which a glass transition has been detected experimentally [8,9,27], thus providing an archetype for quantitative tests of the theory. Comprehensive comparisons between MCT results for the evolution of structural relaxation and the correspond- 
ing experimental findings obtained by dynamic light scattering for hard-sphere colloids were published in Refs. $[8,9,28,29]$. In the data analysis, two numbers enter as fit parameters: a time scale $t_{0}$, given by the viscosity of the solvent, and the critical packing fraction $\varphi_{c}$. The predicted value for $\varphi_{c}$ [2] differs from the experimental value by about $12 \%$. Here, as in any other test of a singularity theory, data have been studied as a function of the distance $\varepsilon=(\varphi$ $\left.-\varphi_{c}\right) / \varphi_{c}$ from the position $\varphi_{c}$ of the singularity. The cited tests have been summarized as follows [30,31]: the leading asymptotic MCT results account for data within the experimental uncertainties for the liquid states $(\varepsilon<0)$ and also for the glass states $(\varepsilon>0)$. This does not only hold for universal formulas like the scaling laws. It also holds for the numerical values predicted for the exponents, the master functions entering the scaling laws, and for the various wave-vectordependent amplitudes. Therefore, it seems justified to illustrate our theory for the tagged-particle motion in a HSS. The cited and tested results for the density dynamics will enter as input taken from the preceding work [18]. But the HSS is also relevant because its dynamics shares many features with other simple systems, as will again be demonstrated in this paper.

The paper is arranged as follows. After briefly reviewing the basics of the MCT glass transition (Sec. II) we start by discussing the properties of the tagged-particle density correlator in Sec. III. Section IV covers the mean-squared displacement and its asymptotic behavior. The quality of the Gaussian approximation for the self-intermediate scattering function of the HSS is assessed (Sec. V), before turning to the non-Gaussian parameter in Sec. VI. In the conclusion we will summarize our results and suggest how some recent experiments and simulations on single-particle quantities can be interpreted.

\section{IDEAL MCT GLASS TRANSITION}

The idealized MCT deals with the structural dynamics of simple liquids, consisting of $N$ particles at positions $\vec{r}_{j}, j=1, \ldots, N$. A self-consistent treatment of the cage effect, which is thought to be the reason for the glass transition, leads to a closed set of integrodifferential equations for the density correlator $\Phi_{q}(t)=\left\langle\rho_{q}^{*}(0) \rho_{q}^{*}(t)\right\rangle / S_{q}$, i.e., the autocorrelation function of the density fluctuation $\rho_{\vec{q}}(t)$ $=(1 / \sqrt{N}) \sum_{j} \exp \left[i \vec{q} \cdot \vec{r}_{j}(t)\right]$ for wave vector $\vec{q}$, normalized by the static structure factor $S_{q}=\left\langle\rho_{q}^{*}(0) \rho_{q}^{*}(0)\right\rangle ;\langle\rangle$ signifies an average with respect to the canonical ensemble, and $q$ $=|\vec{q}|$ abbreviates a wave-vector modulus. Specializing to a colloid model, the MCT equations of motion read

$$
\tau_{q} \dot{\Phi}_{q}(t)+\Phi_{q}(t)+\int_{0}^{t} m_{q}\left(t-t^{\prime}\right) \dot{\Phi}_{q}\left(t^{\prime}\right) d t^{\prime}=0 .
$$

They are specified by the times $\tau_{q}=S_{q} /\left(D_{0} q^{2}\right)$ with $D_{0}$ denoting the single-particle diffusion coefficient. The memory kernel in this equation is given as a mode-coupling functional $m_{q}(t)=\mathcal{F}_{q}(\Phi(t))$, where $\mathcal{F}_{q}$ is determined by the structure factor $S_{q}$ [2], which depends smoothly on external control parameters such as the density $n$.
At a critical density $n_{c}$ the above equations exhibit a bifurcation in the long-time limits $f_{q}=\lim _{t \rightarrow \infty} \Phi_{q}(t)$ of the density correlators. The bifurcation singularity can be identified with a liquid-glass transition: At $n_{c}$, the Debye-Waller factor $f_{q}$, also called the form factor, jumps from zero to the critical from factor $f_{q}^{c}>0$. The instability of the glass for $n$ $\rightarrow n_{c}+$ is reflected by a square-root singularity of the DebyeWaller factor: $f_{q}=f_{q}^{c}+h_{q} \sqrt{\sigma /(1-\lambda)}+O(\sigma)$. Here, $\sigma=C \varepsilon$ is called the separation parameter, $0.5 \leqslant \lambda<1$ is the called exponent parameter, and $h_{q}=\left(1-f_{q}^{c}\right)^{2} e_{q}>0$ denotes the critical amplitude. The quantities $e_{q}, \lambda$, and the constant $C>0$, relating $\sigma$ and the reduced density $\varepsilon=\left(n-n_{c} / n_{c}\right.$, can be calculated by straightforward formulas from the functional $\mathcal{F}[3]$.

Via a simple transcendental equation, the exponent parameter $\lambda$ determines two anomalous exponents: the critical exponent $a, 0<a<0.5$, and the von Schweidler exponent $b, 0<b \leqslant 1$. Furthermore $\lambda$ fixes a constant $B>0$. There appears a single time $t_{0}$, specifying the scale for the transient dynamics. The bifurcation dynamics is then ruled by two critical time scales, denoted by $t_{\sigma}$ and $t_{\sigma}^{\prime}$ :

$$
\begin{gathered}
t_{\sigma}=t_{0} /|\sigma|^{\delta}, \quad t_{\sigma}^{\prime}=t_{0} B^{-1 / b} /|\sigma|^{\gamma}, \\
\delta=\frac{1}{2 a}, \quad \gamma=\frac{1}{2 a}+\frac{1}{2 b} .
\end{gathered}
$$

The mathematical relevance of these concepts is evident from the following limit results [3]. First, $\lim _{\hat{t} \rightarrow 0} \lim _{\sigma \rightarrow 0}\left\{\left[\Phi_{q}\left(\hat{t} t_{\sigma}\right)-f_{q}^{c}\right] / \sqrt{|\sigma|}\right\} \hat{t}^{a}=h_{q}$. Thus, near the transition, the plateau value $f_{q}^{c}$ is approached from above according to a power law, called critical decay,

$$
\Phi_{q}(t)-f_{q}^{c} \sim h_{q}\left(t_{0} / t\right)^{a}, \quad t_{0} \ll t \ll t_{\sigma} .
$$

Second,

$$
\begin{aligned}
& \lim \lim \left[\Phi_{q}\left(\tilde{t} t_{\sigma}^{\prime}\right)-f_{q}^{c}\right] / \widetilde{t}^{b}=-h_{q}=\lim \lim \left\{\left[\Phi_{q}\left(\hat{t} t_{\sigma}\right)\right.\right. \\
& \tilde{t} \rightarrow 0^{\sigma \rightarrow 0-} \quad \hat{t} \rightarrow \infty \sigma \rightarrow 0- \\
& \left.\left.-f_{q}^{c}\right] / \sqrt{|\sigma|}\right\} / \hat{t}^{b} .
\end{aligned}
$$

Near the transition the plateau value $f_{q}^{c}$ is left in the liquid state according to a power law, called von Schweidler decay:

$$
\Phi_{q}(t)-f_{q}^{c} \sim-h_{q}\left(t / t_{\sigma}^{\prime}\right)^{b}, \quad t_{\sigma} \ll t \ll t_{\sigma}^{\prime} .
$$

There are two dynamical scaling laws describing the bifurcation dynamics near the transition in a leading-order asymptotic limit. The first one describes the dynamics on a scale $t_{\sigma}: \Phi_{q}(t)-f_{q}^{c} \sim h_{q} G(t)$. Here $G(t)=\left(t_{0} / t\right)^{a}$ for $\sigma=0$, and

$$
G(t)=\sqrt{|\sigma|} g_{ \pm}\left(t / t_{\sigma}\right), \quad \sigma \gtrless 0 .
$$

The $\sigma$-independent master functions $g_{ \pm}$are determined by $\lambda$. They describe the crossover from the critical decay $g_{ \pm}(\hat{t} \ll 1)=1 / \hat{t}^{a}$ to arrest in the glass, $g_{+}(\hat{t} \gg 1)=1 / \sqrt{1-\lambda}$, or to von Schweidler's decay in the liquid, $g_{-}(\hat{t} \gg 1)$ $=-B \hat{t}^{b}+B_{1} /\left(B \hat{t}^{b}\right)$. The result is obtained by solving the MCT equations using $\left|\Phi_{q}(t)-f_{q}^{c}\right|$ as small parameter. This 
is equivalent to writing $t=\hat{t} t_{\sigma}$ and then expanding in powers of the small parameter $\sqrt{|\sigma|}$. Pushing the expansion to nextto-leading order extends the first scaling-law result to

$$
\Phi_{q}(t)=f_{q}^{c}+h_{q} G(t)+h_{q}\left[H(t)+K_{q} G(t)^{2}+\sigma \hat{K}_{q}\right]
$$

Here two further amplitudes $K_{q}$ and $\hat{K}_{q}$, and a new function $H(t)$, appear. The latter obeys scaling laws $H(t)$ $=\kappa(a)\left(t_{0} / t\right)^{2 a}$ for $\sigma=0$ and $H(t)=|\sigma| h_{ \pm}\left(t / t_{\sigma}\right)$ for $\sigma \gtrless 0$. All these quantities can be evaluated from the modecoupling functional $\mathcal{F}_{q}$ as is comprehensively explained in Ref. [18], where we changed notation $\overline{\bar{K}}$ to $\hat{K}_{q}$. The domain of applicability of expansion (6) is called the $\beta$-relaxation window, and correspondingly $G$ and $t_{\sigma}$ are referred to as the $\beta$ correlator and $\beta$-relaxation time scale, respectively.

The second scaling law describes the liquid dynamics on scale $t_{\sigma}^{\prime}$. It deals with the decay of the correlator from the plateau to zero, and it is based on the relation $\lim _{\sigma \rightarrow 0-} \Phi_{q}\left(\tilde{t} t_{\sigma}^{\prime}\right)=\widetilde{\Phi}_{q}(\tilde{t})$. Here the $\sigma$-independent functions $\widetilde{\Phi}_{q}(\widetilde{t})$ are the solution of the implicit functional equations

$$
\int_{0}^{\tilde{t}}\left[\tilde{m}_{q}\left(t^{\prime}\right)-\tilde{\Phi}_{q}\left(t^{\prime}\right)\right] d t^{\prime}=\int_{0}^{\tilde{t}} \tilde{m}_{q}\left(\tilde{t}-t^{\prime}\right) \tilde{\Phi}_{q}\left(t^{\prime}\right) d t^{\prime}
$$

The kernel $\tilde{m}_{q}(\tilde{t})=\mathcal{F}_{q}^{c}(\tilde{\Phi}(\tilde{t}))$ is the mode-coupling functional at the transition point, and the equation is to be solved with von Schweidler's law as an initial condition. Near the transition within the window $t_{\sigma} \ll t$ this implies, in leading order,

$$
\Phi_{q}(t)=\tilde{\Phi}_{q}\left(t / t_{\sigma}^{\prime}\right)
$$

This window is referred to as the $\alpha$ regime, $t_{\sigma}^{\prime}$ is called the $\alpha$-relaxation time scale, and Eq. (8) is the superposition principle for the $\alpha$ process.

Every theory dealing with the dynamics of a variable $A$ as a probe of structural relaxation in glassy systems relies on an understanding of the dynamics of density rearrangements. Hence a microscopic theory will require results for $\Phi_{q}(t)$ as input. For the following discussion it is therefore necessary to appreciate the concepts and results formulated above. They were explained and illustrated in Ref. [18] for the HSS. The quantitative examples of the following discussion shall also be done for that model. We follow the previous conventions by using the particle diameter $d$ as unit of length, $d$ $=1$, choosing the time unit so that the short-time diffusivity $D_{0}=\frac{1}{160}$, and using the packing fraction $\varphi=\pi n d^{3} / 6$ as the control parameter. In this case one obtains $\varphi_{c}=0.516, C$ $=1.54, \quad \lambda=0.735, \quad a=0.312, \quad b=0.583, B=0.836, B_{1}$ $=0.431$, and $t_{0}=0.425$.

\section{TAGGED-PARTICLE DENSITY CORRELATOR}

\section{A. Equation of motion}

The dynamics of a tagged particle is explored by its density correlator $\Phi_{q}^{s}(t)=\left\langle\rho_{\vec{q}}^{s *}(0) \rho_{q}^{s}(t)\right\rangle, \quad$ where $\quad \rho_{\vec{q}}^{s}(t)$ $=\exp [i \vec{q} \cdot \vec{r}(t)] ; \vec{r}$ denotes the position of the particle. For a system driven by Brownian dynamics the MCT equation of motion reads

$$
\tau_{q}^{s} \dot{\Phi}_{q}^{s}(t)+\Phi_{q}^{s}(t)+\int_{0}^{t} m_{q}^{s}\left(t-t^{\prime}\right) \dot{\Phi}_{q}^{s}\left(t^{\prime}\right) d t^{\prime}=0,
$$

with $\tau_{q}^{s}=1 /\left(D_{0}^{s} q^{2}\right)$. Here $D_{0}^{s}$ is the short-time diffusion coefficient of the immersed particle. The memory kernel $m_{q}^{s}(t)=\mathcal{F}_{q}^{s}\left(\Phi(t), \Phi^{s}(t)\right)$ is expressed through the modecoupling functional $\mathcal{F}_{q}^{s}$, where not only $\Phi_{q}^{s}(t)$ but also $\Phi_{q}(t)$ enter:

$$
\mathcal{F}_{q}^{s}\left(f, f^{s}\right)=\frac{1}{(2 \pi)^{3}} \int n S_{k} c_{k}^{s 2}\left(\frac{\vec{q} \vec{k}}{q^{2}}\right)^{2} f_{k} f_{|\vec{q}-\vec{k}|}^{s} d^{3} k
$$

Here $c_{q}^{s}=\left\langle\rho_{q}^{s *}(0) \rho_{q}^{\vec{q}}(0)\right\rangle /\left(n S_{q}\right)$ denotes the single-particle direct correlation function [2].

To solve Eqs. (9) and (10) numerically, we proceed as in Ref. [18]: The mode-coupling functional is rewritten in bipolar coordinates by change of variable, and the two remaining integrals are approximated by Riemann sums

$$
\begin{aligned}
\mathcal{F}_{q}^{s}\left(f, f^{s}\right)= & n \Delta^{3} /\left(16 d^{3} \pi^{2}\right) \sum_{\hat{k}=1 / 2}^{99.5} \sum_{\hat{p}=1 / 2}^{99.5} S_{k}\left(\hat{k} \hat{p} / \hat{q}^{5}\right)\left(\hat{k}^{2}+\hat{q}^{2}\right. \\
& \left.-\hat{p}^{2}\right)^{2} c_{k}^{s 2} f_{k} f_{p}^{s}
\end{aligned}
$$

The prime indicates that the sum runs only over those values $\hat{p}$ for which $\hat{k}, \hat{p}$, and $\hat{q}$ obey the triangle inequality. Thereby, we again obtain a precisely defined tagged-hardsphere-particle model described by 100 coupled integrodifferential equations for $\Phi_{q}^{s}(t)$ on a grid of 100 equally spaced wave numbers $q=\Delta \hat{q}$ with $\hat{q}=\frac{1}{2}, \ldots, 99 \frac{1}{2}$ and step size $\Delta=0.4$.

The substitution of Eq. (11) for the mode-coupling functional (10) might seem rather crude. However, we have checked that the results seldom differ by more than a few percent from those obtained with 300 or 900 grid points for the Riemann sum. Only at small wave numbers do qualitative changes occur due to bad angle resolution; however, these do not influence other wave vectors because the major contributions to the integral in Eq. (10) come from the wave vectors near the structure-factor-peak position.

In our application to the HSS, the static structure factor $S_{q}$ and the direct correlation function $c_{q}^{s}$ are calculated in the Percus-Yevick approximation [32]. Assuming short-time diffusion according to Stokes' law, which entails $D_{0}^{s} / D_{0}$ $=d / d^{s}$, only two control parameters remain: the packing fraction $\varphi$ of the host particles and the diameter $d^{S}$ of the tagged particle. All results for the coherent density correlators $\Phi_{q}(t)$ of the HSS, which will be needed in the following, will be taken from the solution of Eq. (1) discussed in Ref. [18]. 


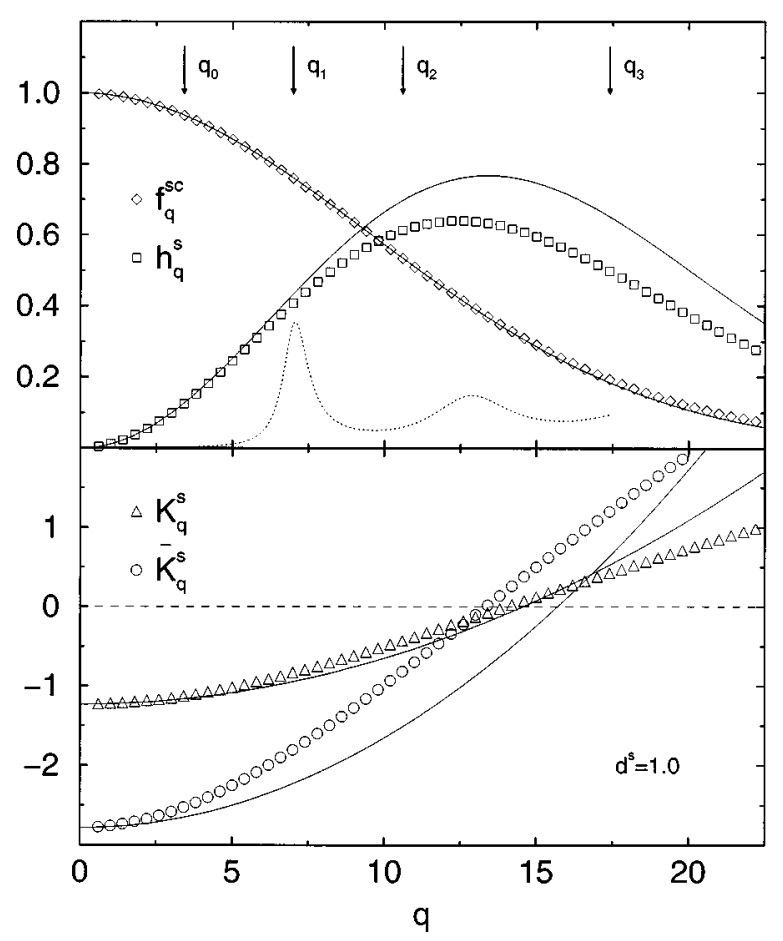

FIG. 1. Critical Lamb-Mössbauer factor $f_{q}^{s c}$ and amplitudes $h_{q}^{s}, K_{q}^{s}$, and $\bar{K}_{q}^{s}$, obtained from Eqs. (12), (15), and (17), for a tagged hard-sphere particle of diameter $d^{s}=1.0$ immersed in a hardsphere liquid. The solid lines are the Gaussian approximations, Eqs. (31). The arrows indicate the wave numbers $q_{0}=3.4, \quad q_{1}$ $=7.0, q_{2}=10.6$, and $q_{3}=17.4$. The dotted line in the upper panel is one tenth of the structure factor at the critical point (compare Fig. 3 in Ref. [18]). Here and in the following figures the diameter $d$ of the host particles is chosen as the unit of length.

\section{B. Critical Lamb-Mössbauer factor}

The trapping of a tagged particle by its surrounding host particles manifests itself by a nonvanishing LambMössbauer factor $f_{q}^{s}=\lim _{t \rightarrow \infty} \Phi_{q}^{s}(t)$. From Eq. (9), one finds [2] that $f_{q}^{s}$ is a solution of

$$
\frac{f_{q}^{s}}{1-f_{q}^{s}}=\mathcal{F}_{q}^{s}\left(f, f^{s}\right)
$$

It is distinguished from other solutions of Eq. (12), say $\widetilde{f}_{q}^{s}$, by the maximum property, $f_{q}^{s} \geqslant \widetilde{f}_{q}^{s}$ [3]. It can be found by the iteration $f_{q}^{s}=\lim _{n \rightarrow \infty} f_{q}^{s(n)}$, where $f_{q}^{s(n+1)} /\left(1-f_{q}^{s(n+1)}\right)$ $=\mathcal{F}_{q}^{s}\left(f, f^{s(n)}\right), f_{q}^{s(0)}=1$ [33]. Obviously $f \equiv 0$ implies $f^{s}$ $\equiv 0$, but not vice versa. The spatial Fourier transform $f^{s}(r)$ of the Lamb-Mössbauer factor is the probability of finding the particle for $t=\infty$ at distance $r$ from where it started for $t=0$. Therefore, a particle cannot be trapped as long as the host particles are in a liquid state. But even when the host particles are in a glass state, the tagged particle may still be able to diffuse in the arrested structure. The various scenarios arising from Eqs. (12) and (10) have been discussed before (compare Ref. [34] and the papers cited there).

For a tagged hard sphere of diameter $d^{s}=1.0$ the LambMössbauer factor jumps from 0 to the critical value $f_{q}^{s c}>0$, which is shown in Fig. 1, when the packing fraction $\varphi$ passes

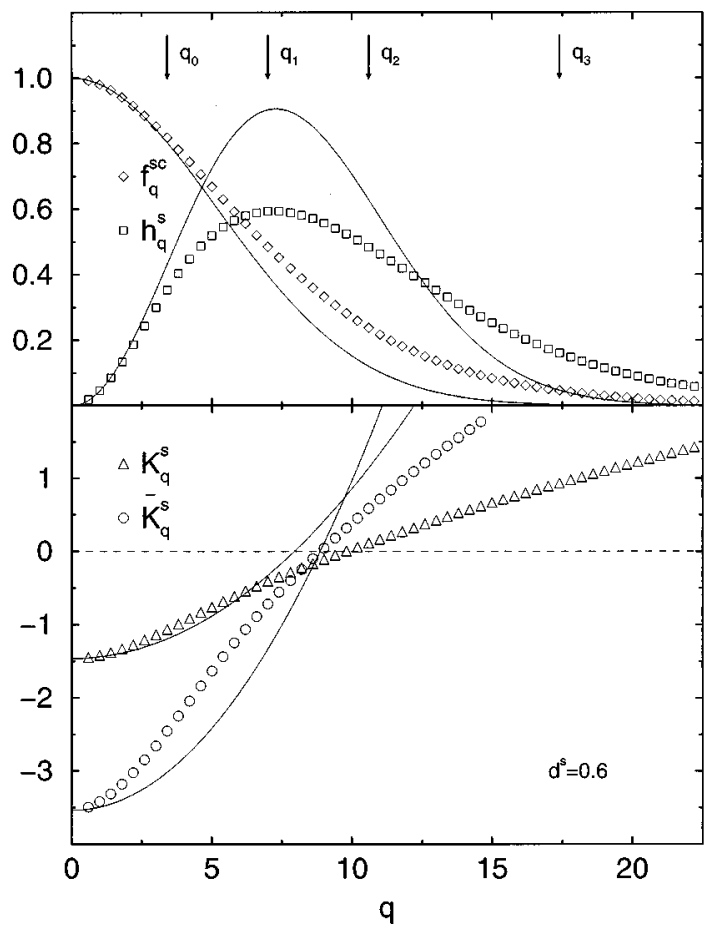

FIG. 2. Same as Fig. 1 for a tagged particle of diameter $d^{s}$ $=0.6$.

through the glass-transition singularity $\varphi_{c}$. For $d^{s}=0.6$ the particle still becomes trapped at $\varphi_{c}$, but the $f_{q}^{s c}$-versus- $q$ graph is narrower than before (see Fig. 2). This means that the center of the smaller particle can explore a larger volume. Reducing the particle size $d^{s}$ further, the system passes through a percolation threshold at some critical diameter $d^{s c}$. In the following we will only be interested in tagged particles with diameters $d^{s}>d^{s c}$, for which the LambMössbauer factor shows the generic fold bifurcation.

\section{Asymptotic laws}

\section{1. $\beta$ relaxation}

Close to the bifurcation, the correlators $\Phi_{q}^{s}(t)$ show a characteristic two-step relaxation process, as can be seen in Fig. 3. In Ref. [4] it was discussed, and in Ref. [35] it was demonstrated for relevant examples, that the long-time behavior of correlators, which are the solutions of MCT equations such as Eqs. (1) and (9), is determined by

$$
\frac{\Phi_{q}^{s}(s)}{1-s \Phi_{q}^{s}(s)}=\mathcal{L}\left[\mathcal{F}_{q}^{s}\left(\Phi(t), \Phi^{s}(t)\right)\right](s) .
$$

Here $\Phi_{q}^{s}(s)$ denotes the Laplace transform $\Phi(s)$ $=\mathcal{L}[\Phi(t)](s):=\int_{0}^{\infty} e^{-s t} \Phi(t) d t$ of $\Phi_{q}^{s}(t)$. These equations depend only on equilibrium quantities via the static structure factor. They are therefore independent of the microscopic dynamics and any additional regular contributions to the kernels $m_{q}(t)$ and $m_{q}^{s}(t)$. Consequently, it makes no difference for the asymptotic results discussed throughout this paper that we chose Brownian instead of Newtonian dynamics, and omitted regular contributions to the kernels. 


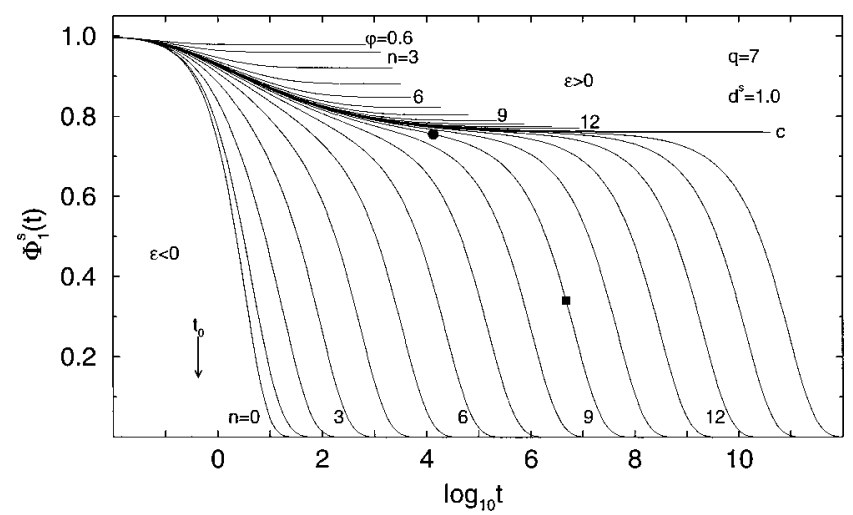

FIG. 3. Self-intermediate scattering function $\Phi_{1}^{s}(t)$, calculated from Eqs. (9) and (11), of the HSS $\left(d^{s}=1.0\right)$ for wave number $q_{1}=7.0$ at various reduced packing fractions $\varepsilon=\left(\varphi-\varphi_{c}\right) / \varphi_{c}$ $= \pm 10^{-n / 3}$, where $n=0, \ldots, 14$. The thick curve labeled $c$ shows the dynamics at the critical packing fraction $\varphi_{c}=0.515912 \ldots$. The uppermost curve refers to the packing fraction $\varphi=0.6$. The arrow marks the time $t_{0}=0.425$. Here and in some of the following figures the full dot and square mark the times $t_{\sigma}$ and $t_{\sigma}^{\prime}$, respectively, for $\varepsilon=-0.001$. On the glass side $(\varepsilon>0)$ the curves for $n$ $=13$ and 14 are omitted since they would be barely distinguishable from curve $c$. The $n=0,1$, and 2 glass curves are omitted, since the Percus-Yevick approximation produces negative values for the pair distribution function for such high densities. Here and in the following figures the unit of time is chosen such that the short-timediffusion coefficient of the host particles reads $D_{0}=\frac{1}{160}$.

Inserting the asymptotic expansion (6) into Eq. (13), using Taylor's theorem on the mode-coupling functional $\mathcal{F}_{q}^{s}$, and collecting like-order terms, one obtains, in leading and nextto-leading order,

$$
\Phi_{q}^{s}(t)=f_{q}^{s c}+h_{q}^{s} G(t)+h_{q}^{s}\left[H(t)+K_{q}^{s} G(t)^{2}+\sigma \hat{K}_{q}^{s}\right] .
$$

Here the critical amplitude $h_{q}^{s}=\left(1-f_{q}^{s c}\right)^{2} e_{k}^{s}$ for the taggedparticle correlator and the correction amplitudes $K_{q}^{s}$ and $\hat{K}_{q}^{s}$ are to be calculated from

$$
\begin{gathered}
\sum_{k}\left(\delta_{q k}-C_{q k}^{s c}\right) e_{k}^{s}=\sum_{k} C_{q, k}^{s c} e_{k} \\
\sum_{k}\left(\delta_{q k}-C_{q k}^{s c}\right) e_{k}^{s} K_{k}^{s}=-\left(1-f_{q}^{s c}\right) e_{q}^{s 2} \lambda+\sum_{k} C_{q, k}^{s c} e_{k} K_{k} \\
+\sum_{p, k} C_{q p k}^{s c} e_{p}^{s} e_{k}^{s}+\sum_{p, k} C_{q, p k}^{s c} e_{p} e_{k} \\
+\sum_{p, k} C_{q p, k}^{s c} e_{p}^{s} e_{k} \\
\sum_{k}\left(\delta_{q k}-C_{q k}^{s c}\right) e_{k}^{s} \hat{K}_{k}^{s}=-\left(1-f_{q}^{s c}\right) e_{q}^{s 2}+\delta C_{q}^{s} / C \\
+\sum_{k} C_{q, k}^{s c} e_{k} \hat{K}_{k} .
\end{gathered}
$$

Taylor coefficients of the mode-coupling functional are introduced by

$$
\begin{aligned}
C_{q p_{1} \cdots p_{n}, k_{1} \cdots k_{m}}^{s}= & C_{q p_{1} \cdots p_{n}, k_{1} \cdots k_{m}}^{s c} \\
& +\delta C_{q p_{1} \cdots p_{n}, k_{1} \cdots k_{m}}^{s} \varepsilon+O\left(\varepsilon^{2}\right) \\
= & \frac{1}{n !} \frac{1}{m !} \frac{\partial^{n} \partial^{m} \mathcal{F}_{q}^{s}\left(f^{c}, f^{s c}\right)}{\partial f_{p_{1}} \cdots \partial f_{p_{n}} \partial f_{k_{1}} \cdots \partial f_{k_{m}}} \\
& \times\left(1-f_{p_{1}}^{s c}\right)^{2} \cdots\left(1-f_{p_{n}}^{s c}\right)^{2} \\
& \times\left(1-f_{k_{1}}^{c}\right)^{2} \cdots\left(1-f_{k_{m}}^{c}\right)^{2} .
\end{aligned}
$$

For the derivation of these general formulas, we made no use of the linearity of the mode-coupling functional in $f$ and $f^{s}$. Figures 1 and 2 exhibit the HSS results for the amplitudes for the two diameters $d^{s}=1.0$ and $d^{s}=0.6$, respectively. Following Ref. [18] we show

$$
\bar{K}_{q}^{s}=\hat{K}_{q}^{s} \sqrt{1-\lambda}+K_{q}^{s} / \sqrt{1-\lambda}-\kappa+h_{+}(\infty) \sqrt{1-\lambda}
$$

instead of $\hat{K}_{q}^{s}$, where $\kappa=0.961$ and $h_{+}(\infty)=-2.21$ for the HSS.

Equation (14) for the tagged-particle correlator is formally identical to the above expansion (6) for the coherent density correlator. This reflects the universality of the asymptotic features of the MCT. Within the $\beta$-relaxation window the dynamics is fixed by the $\beta$ correlator $G(t)$ in leading order, and by $G$ and $H$ in next-to-leading order in an asymptotic expansion. Correlators referring to different probing variables merely differ in the various amplitudes such as $\left(f_{q}^{c}, f_{q}^{s c}\right),\left(h_{q}, h_{q}^{s}\right)$, etc. These amplitudes do not depend on time or on control parameters. However, there are rather important differences for the procedure to determine the amplitudes reflecting the fact that the bifurcation in the taggedparticle dynamics is brought about by the bifurcation in the host-particle dynamics. The amplitudes $e_{q}, K_{q}$ and $\hat{K}_{q}$, for the cage-forming particles are the solutions of singular matrix equations and the solubility conditions in second and third orders of the asymptotic expansion determine the scaling functions $G$ and $H$ up to a single time scale $t_{0}$, which has to be matched at the critical point [18]. By contrast, the positive matrix $C_{q k}^{s c}$ has a spectral radius smaller than unity [33]. Hence, the matrix $\delta_{q k}-C_{q k}^{s c}$ is regular, and the amplitudes are uniquely determined by solving the linear equations (15). The scaling functions $G$ and $H$ are solely determined by the properties of the host particles. The interaction potential of the tagged particle enters the Lamb-Mössbauer factor, and the amplitudes via the direct correlation function $c_{q}^{s}$, which is hidden in the Taylor coefficients (16). No further time scale needs to be fixed. Even a change of the microscopic time scales $\tau_{q}^{s}$ in Eq. (9) would not affect the time scale of the asymptotic behavior. One can always go so close to the critical point that Eq. (14) holds within a prescribed error margin.

The first two terms on the right-hand side of Eq. (14) are known as the factorization theorem: If the density correlators are rescaled accordingly, the curves collapse onto the $\beta$ correlator $G$ in an intermediate time window, the so-called 


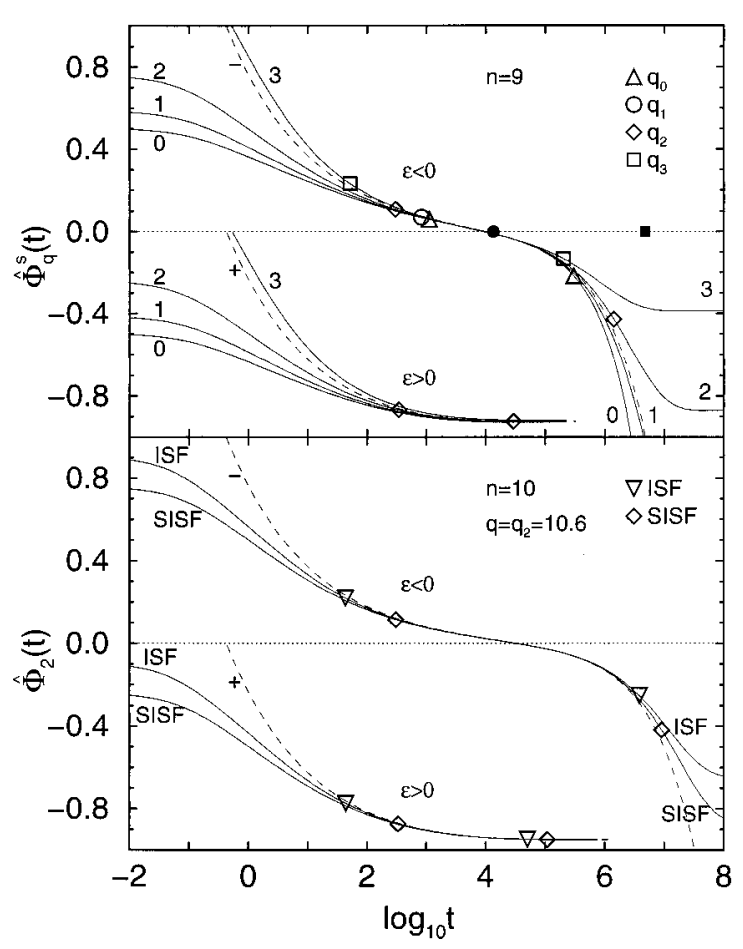

FIG. 4. Upper panel: Rescaled self-intermediate scattering function $\hat{\Phi}_{q}^{s}(t)=\left(\Phi_{q}^{s}(t)-f_{q}^{s c}\right) / h_{q}^{s}$ (solid lines) of the hard-sphere system $\left(d^{s}=1.0\right)$ for four different wave numbers at reduced packing fraction $\varepsilon=\left(\varphi-\varphi_{c}\right) / \varphi_{c}= \pm 10^{-n / 3}$ with $n=9$, showing collapse onto the $\beta$ correlator $G$ [dashed lines, Eq. (5)] in the $\beta$-relaxation region. On the extreme left and right, small numbers near each curve indicate the wave numbers $q_{0}, q_{1}, q_{2}$, and $q_{3}$, marked in Fig. 1 . Lower panel: Rescaled intermediate scattering function (ISF) and self-intermediate scattering function (SISF) for wave number $q_{2}$ $=10.6$ at $n=10$, compared to the $\beta$ correlator $G$. The glass curves $(\varepsilon>0)$ are shifted downward by 1 to avoid overcrowding. The open symbols mark the boundaries of the $\beta$-relaxation region as defined at the end of Sec. III C 1. Some symbols are missing because they are either outside the display range (the long-time part of the liquid curve for $q_{1}$ ) or the range of validity is nonexistent (glass curves for $q_{0}, q_{1}$, and $q_{3}$ ).

$\beta$-relaxation region, as demonstrated in Fig. 4 for four wave numbers. Obviously, the $\beta$-relaxation region depends on the wave number (upper panel) or-put more generally—on the quantity under consideration (lower panel). This is accounted for by the two $q$-dependent amplitudes $K_{q}^{s}$ and $\hat{K}_{q}^{s}$ occurring in the leading correction to the factorization property. The $\beta$ window expands as the system moves closer to the critical point, as demonstrated by a comparison of $\Phi_{q}^{s}(t)$ for $q_{2}$ $=10.6$ for $n=9$ and 10 in Fig. 4. More can be said if we consider that the dominant corrections are the short-time corrections to the critical law (3), and the long-time corrections to the von Schweidler law (4) or the corrections to the LambMössbauer factor $f_{q}^{s}$ on the liquid $(\varepsilon<0)$ or glass $(\varepsilon>0)$ side, respectively. The short-time expansion of the righthand side of Eq. (14) is given by $f_{q}^{s c}+h_{q}^{s}\left(t / t_{0}\right)^{-a}\{1$ $\left.+\left[\kappa(a)+K_{q}^{s}\right]\left(t / t_{0}\right)^{-a}\right\}$, so that the factor $\kappa(a)+K_{q}^{s}$ determines the sign and strength of the leading correction to the critical decay. For the HSS one obtains $\kappa(a)=-0.002$ [18] and $K_{q}^{s}<K_{p}^{s}$ for $q<p$ (compare Fig. 1). This explains in particular why at early times the curves with smaller wave number fall below the curves with larger wave number. Since $\kappa(a)+K_{q}^{s}$ changes sign between $q_{2}$ and $q_{3}$, curves 0 , 1 , and 2 fall below and curve 3 is above the dashed asymptote for $t<10$. On the liquid side the analogous formula $\Phi_{q}^{s}(t)=f_{q}^{s c}-h_{q}^{s} \tilde{t}^{b}\left\{1-\left[\kappa(-b)+K_{q}^{s}\right] \tilde{t}^{b}\right\}$ provides the explanation for the deviations from the von Schweidler asymptote [Eq. (4)] at later times: the $q$ dependence of the deviations is again determined by the amplitude $K_{q}^{s}$. Thus if one curve lies above the other when deviating from the factorization theorem at short times, it will also do so at long times. This rule, which was already discussed for $\Phi_{q}(t)$ in Ref. [18], holds for all quantities that asymptotically obey Eq. (14). For the HSS one obtains $\kappa(-b)=0.569$ [18], and therefore Fig. 1 implies that $\kappa(-b)+K_{q}^{s}$ changes sign between $q_{1}$ and $q_{2}$. This explains why curves 0 and 1 are below, and curves 2 and 3 above, the dashed asymptote in Fig. 4 for $\log _{10} t \approx 6$. On the glass side the long-time behavior of $\Phi_{q}^{s}(t)$ is described by $f_{q}^{s}=f_{q}^{s c}+h_{q}^{s} \sqrt{\sigma /(1-\lambda)}\left[1+\sqrt{\sigma}\left(\bar{K}_{q}^{s}+\kappa\right)\right]$.

Armed with Fig. 2 and what was explained for $d^{s}=1$, it is easy to infer what Fig. 4 would look like for a hard sphere of diameter $d^{s}=0.6$. Since it was already explained in Ref. [18] how the deviations from the leading order translate into the frequency domain, spectra shall not be discussed in this paper.

In order to discuss the quality of the asymptotic expansions in a controllable manner, some convention for the error margin has to be made. There are various possibilities [18]. In this paper the range of validity shall be defined as the time window where, for a given value $\Phi$ of the correlator, the time $t_{\Phi}$ for the solution, $\Phi=\Phi\left(t_{\Phi}\right)$, deviates from the time $t_{\Phi}^{a s}$ for the asymptotic expansion, say $\Phi=f_{q}^{c}+h_{q} G\left(t_{\Phi}^{a s}\right)$, by less than $\tilde{\varepsilon}$ percent. This definition can always be applied to monotonic functions of time, in particular to functions in the $\beta$-relaxation window. If one chooses $\tilde{\varepsilon}$ so small that the deviations from the leading order are quantitatively explained by the leading corrections, the range of validity for the leading-order $\beta$-relaxation asymptote can be shown to expand with the $\beta$ time scale $t_{\sigma}$ for $\sigma \rightarrow 0+$, and with the $\alpha$ time scale $t_{\sigma}^{\prime}$ for $\sigma \rightarrow 0-$. In Fig. 4 and some of the following figures, the end points of the time intervals with $\tilde{\varepsilon}$ $=20 \%$ are marked by various symbols.

\section{2. $\alpha$ relaxation}

Within the $\alpha$-relaxation window, the tagged-particle correlator $\Phi_{q}^{s}(t)$ obeys the superposition principle as formulated in Eq. (8) for the density correlator: $\Phi_{q}^{s}(t)=\widetilde{\Phi}_{q}^{s}\left(t / t_{\sigma}^{\prime}\right)$. The control-parameter-independent master function $\widetilde{\Phi}_{q}^{s}(\widetilde{t})$ is obtained from Eq. (7), where only superscripts $s$ have to be added to correlators and kernels [3].

In Fig. 5, various rescaled correlators $\Phi_{1}^{s}(t)$ are compared to the $\alpha$ master function. The superposition principle best describes the $\alpha$ relaxation for later times. As the glass transition is approached from the liquid side, the range of validity of the superposition principle, which is marked by diamonds and defined in analogy to the $\beta$-relaxation regime above, extends to earlier rescaled times $\tilde{t}$. Close to the critical point, the deviations from the $\alpha$ master curve can be understood in terms of the leading-order correction $\delta \widetilde{\Phi}_{q}^{s}(\tilde{t})$ 


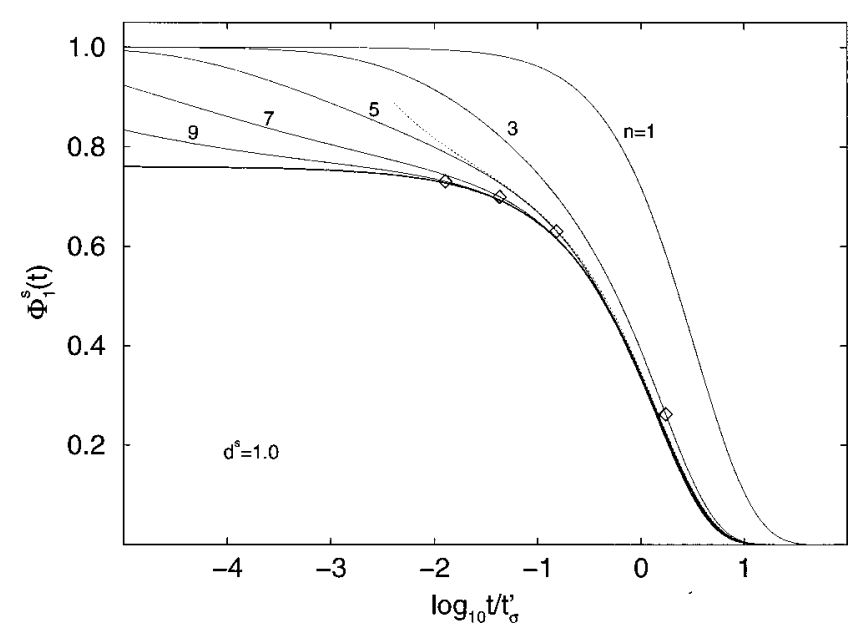

FIG. 5. $\alpha$-scaling plot of the self-intermediate scattering function: $\Phi_{1}^{s}(t)$, taken from Fig. 3, for reduced packing fractions $\varepsilon$ $=\left(\varphi-\varphi_{c}\right) / \varphi_{c}=-10^{-n / 3}, n=1,3,5,7$, and 9 , vs rescaled time $\tilde{t}$ $=t / t_{\sigma}^{\prime}$. The thick solid line is the $\alpha$ master function $\tilde{\Phi}_{1}^{s}(\tilde{t})$. The diamonds mark the early-time bound for the $\alpha$-scaling regime as defined at the end of Sec. III C 1 . The dotted line indicates $\widetilde{\Phi}_{1}^{s}(\tilde{t})$ $-\sigma h_{1} B_{1} \tilde{t}^{b}$, which includes the small- $\tilde{t}$ correction according to Eq. (18).

to the $\alpha$-scaling law, which is linear in $\sigma: \Phi_{q}^{s}(t)=\widetilde{\Phi}_{q}^{s}(\tilde{t})$ $+\delta \tilde{\Phi}_{q}^{s}(\tilde{t})+O\left(\sigma^{2}\right)$ [18]. Although the equation governing the time evolution of $\delta \tilde{\Phi}_{q}^{s}(\tilde{t})$ is rather involved, its shorttime behavior can be expressed in terms of the amplitudes introduced above in connection with the $\beta$ relaxation. Up to errors of order $\sigma \widetilde{t}^{b}$, one obtains

$$
\delta \tilde{\Phi}_{q}^{s}(\tilde{t})=-\sigma h_{q}^{s}\left\{B_{1} \tilde{t}^{-b}+\left[\tilde{\kappa}(-b)-2 B_{1} K_{q}^{s}-\hat{K}_{q}^{s}\right]\right\}
$$

with $\tilde{\kappa}(-b)=2.97$ for the HSS [18]. The $\tilde{t}^{-b}$ term, which diverges for $\tilde{t} \rightarrow 0$, dominates the deviations from $\alpha$ scaling for small $|\sigma|$. Consequently, asymptotically close to $\varphi_{c}$, the deviations occur at short times, where the $\alpha$ master function is described by von Schweidler's law (4). Therefore, the $\tilde{t}$ window for the $\alpha$ scaling expands to smaller rescaled times proportional to $|\sigma|^{1 / 2 b}$. For small $|\sigma|$, the $\alpha$-relaxation window becomes independent of the correction amplitudes $K_{q}^{s}$ and $\hat{K}_{q}^{s}$, and thus independent of the observable under consideration, because the observable-dependent amplitude $h_{q}^{s}$ occurs as a prefactor to both von Schweidler's law and the relevant correction $\tilde{t}^{-b}$. The dotted line in Fig. 5 illustrates how the deviations $\delta \tilde{\Phi}_{q}^{s}(\tilde{t})$ from the $\alpha$ master function can be described by the leading correction $-\sigma h_{q}^{s} B_{1} \tilde{t}^{-b}$.

\section{MEAN-SQUARED DISPLACEMENT}

\section{A. Equation of motion}

The equation of motion for the mean-squared displacement (MSD) $\delta r^{2}(t)=\left\langle|\vec{r}(t)-\vec{r}(0)|^{2}\right\rangle$ [36] can be obtained from Eq. (9) by exploiting its relation to the small-wavenumber behavior of the tagged-particle density correlator $\Phi_{q}^{s}(t)=1-q^{2} \delta r^{2}(t) / 6+O\left(q^{4}\right)$ :

$$
\delta r^{2}(t)+D_{0}^{s} \int_{0}^{t} m^{(0)}\left(t-t^{\prime}\right) \delta r^{2}\left(t^{\prime}\right) d t^{\prime}=6 D_{0}^{s} t
$$

Here we have introduced the kernel $m^{(0)}(t)$ $=\lim _{q \rightarrow 0} q^{2} m_{q}^{s}(t)$. Carrying out the limit $q \rightarrow 0$ in Eq. (10), one finds the representation of the new kernel as a new mode-coupling functional, $m^{(0)}(t)=\mathcal{F}_{\mathrm{MSD}}\left(\Phi(t), \Phi^{s}(t)\right)$, where

$$
\mathcal{F}_{\mathrm{MSD}}\left(f, f^{s}\right)=\frac{1}{6 \pi^{2}} \int_{0}^{\infty} n S_{k} c_{k}^{s 2} k^{4} f_{k} f_{k}^{s} d k
$$

Again this integral shall be rewritten as a Riemann sum over the previously introduced wave-vector grid of 100 terms:

$$
\mathcal{F}_{M S D}\left(f, f^{s}\right)=\left[n \Delta^{5} /\left(6 \pi^{2}\right)\right] \sum_{\hat{k}=1 / 2}^{99.5} S_{k} c_{k}^{s 2} \hat{k}^{4} f_{k} f_{k}^{s}
$$

Two side remarks concerning the preceding formulas might be of interest. First, instead of solving Eq. (19) with $\Phi_{q}(t)$ and $\Phi_{q}^{s}(t)$ as an input for the determination of the kernel $m^{(0)}$, one could obtain the desired result directly as the small-wave-vector limit $\delta r^{2}(t)=6 \lim _{q \rightarrow 0}\left[1-\Phi_{q}^{s}(t)\right] /$ $q^{2}$. We have studied this procedure, and checked the result to be the same as obtained from Eqs. (19) and (20). However, to do this, we had to use more than 100 grid points for the Riemann sum, and extrapolate carefully from small- $q$ to zero. This is necessary, since the discretization in Eq. (11) is too crude to produce reliable results for very small $q$, say $q=\Delta$ or $2 \Delta$. However, after carrying out the small $q$ limit for $q^{2} m_{q}^{s}(t)$, the discretization (21) of the integral in Eq. (20) is harmless since the major contributions come from the intermediate wave-vector domain $k \sim 7$. This reflects the fact that the sluggish dynamics of the tagged particle is ruled by the cage effect, i.e., by structure correlations on length scales of the interparticle distance.

Second, there is a trivial relation between $\delta r^{2}(t)$ and the velocity correlation function $K^{s}(t)$. Equations (19) and (20) are equivalent to the MCT equation discussed earlier for $K^{s}(t)$ in the Laplace domain [3]. Following this route, $\delta r^{2}(t)$ has been evaluated previously for some representative packing fractions for the HSS [26]. We prefer to solve Eq. (19) directly, since thereby we need not worry about a careful handling of the strong small-s divergency exhibited by $\mathcal{L}\left[\delta r^{2}(t)\right](s)$.

\section{B. Diffusion-localization transition}

In order to understand that the ideal liquid-glass transition implies a transition from particle localization to particle diffusion, let us remember that the Fourier back transform $\Phi^{s}(r, t)=\langle\delta(\vec{r}-[\vec{r}(t)-\vec{r}(0)])\rangle$ of the tagged-particle density correlator from the wave vector to the displacement domain is the probability density for finding at time $t$ the particle in a distance $r$ from its starting position. In the glass this distribution levels off for long times at the normalized distribution $f^{s}(r)$. Hence the tagged particle is localized in the glass matrix. A characteristic localization length $r_{s}$ can be defined, for example, in terms of the small- $q$ limit of the 


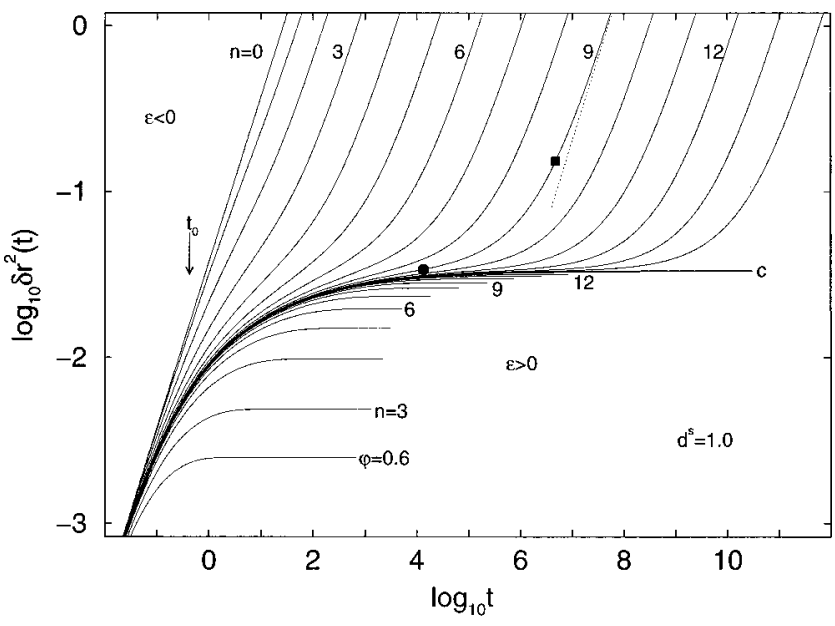

FIG. 6. Mean-square displacement $\delta r^{2}(t)$, obtained from Eqs. (19) and (21), for the hard-sphere system $\left(d^{s}=1.0\right)$ at various packing fractions. The solid curves are labeled as in Fig. 3. The straight dotted line with unit slope indicates the long-time-diffusion asymptote $\left(6 D^{s} t\right)$ for the $n=9$ liquid curve.

Lamb-Mössbauer factor: $f_{q}^{s}=1-\left(q r_{s}\right)^{2}+O\left(q^{4}\right)$. This fixes the long-time asymptote of the mean-squared displacement for $\sigma>0$ :

$$
\lim _{t \rightarrow \infty} \delta r^{2}(t)=6 r_{s}^{2} .
$$

Obviously, $\quad \delta r^{2}(t)=\int \Phi^{s}(r, t) r^{2} d^{3} r, \quad$ so that $6 r_{s}^{2}$ $=\int f^{s}(r) r^{2} d^{3} r$. Equation (19) yields the general formula for $r_{s}$ in terms of sums over the glass form factors [3]

$$
r_{s}^{2}=1 / \mathcal{F}_{\mathrm{MSD}}\left(f, f^{s}\right) .
$$

In the liquid state the low-frequency behavior of the Laplace transform of all correlation functions and kernels is smooth [33]. Therefore, from Eq. (19) one derives the well known formula for the linear divergency in time:

$$
\lim _{t \rightarrow \infty} \delta r^{2}(t) / t=6 D^{s} .
$$

Here $D^{s}$ is the tagged-particle diffusivity. It is also called the long-time diffusivity in order to distinguish it from $D_{0}^{s}$, which determines the short-time asymptote $\lim _{t \rightarrow 0} \delta r^{2}(t) / t$ $=6 D_{0}^{s}$. From Eq. (19) one readily derives for the ratio of $D^{s}$ and $D_{0}^{s}$ :

$$
\frac{D^{s}}{D_{0}^{s}}=\frac{1}{1+D_{0}^{s} \int_{0}^{\infty} m^{(0)}(t) d t}
$$

Note that this ratio is less than unity, which is an obvious manifestation of the cage effect.

Figure 6 exhibits the evolution of the bifurcation dynamics as probed by $\delta r^{2}(t)$ for the HSS. For very small $t$, one observes short-time diffusion. With increasing density the short-time diffusion is suppressed due to the cage effect. The glass curves level off for long times at $6 r_{s}^{2}$. With decreasing density the localization length increases up to some critical value $r_{s c}$. The critical value $r_{s c}=0.0746$ fits nicely to the Lindemann melting criterion, as already noted in Ref. [2]. The liquid curves intersect the plateau $r_{s c}$ for times of order $t_{\sigma}$; the time $t_{\sigma}$ is indicated for the $n=9$ curve by a dot in Fig. 6. Then they leave the plateau according to von Schweidler's law in order to cross over to the diffusion limit $\delta r^{2}(t)=6 D^{s} t$ for times large compared to $t_{\sigma}^{\prime}$. For the $n$ $=9$ curve, the latter asymptote is indicated by a dotted line in Fig. 6, and the time $t_{\sigma}^{\prime}$ is marked by a full square on the graph. The increase of $\delta r^{2}(t)$ above $6 r_{s}^{2}$ is the $\alpha$ process of the mean-squared-displacement dynamics. It deals with the tagged particle's leaving of the cage. The initial part of this process, where $\delta r^{2}(t)$ is close to $r_{s c}^{2}$, is stretched over a large dynamical window if $|\varepsilon|$ is small. For times of order $20 t_{\sigma}^{\prime}, \delta r^{2}(t)$ has increased to about unity; then stochastic dynamics sets in and $\delta r^{2}(t)$ follows the diffusion asymptote. Between the end of the regular short-time transient and the start of the $\alpha$ process, there is a mesoscopic window for another anomalous dynamics. It deals with the stretched approach of $\delta r^{2}(t)$ toward the plateau $6 r_{s}^{2}$. In this sense the bifurcation dynamics, i.e., the dynamics outside the transient, deals with a two-step relaxation process. Asymptotic expansions shall be used to describe the bifurcation scenario by analytical formulas, thereby providing an understanding of Fig. 6.

\section{Asymptotic laws}

\section{1. $\beta$ relaxation}

An equation connecting the Laplace transforms $\delta r^{2}(s)$ and $m^{(0)}(s)$ of $\delta r^{2}(t)$ and $m^{(0)}(t)$, respectively, within the structural relaxation regime $t \gg t_{0}$, follows from Eq. (13): $s \delta r^{2}(s)=6 /\left[s m^{(0)}(s)\right]$. By inserting the asymptotic expansions (6) and (14) into this equation, one again obtains the general result for the $\beta$ relaxation to next-to-leading order:

$$
\begin{aligned}
\delta r^{2}(t) / 6= & r_{s c}^{2}-h_{\mathrm{MSD}} G(t) \\
& -h_{\mathrm{MSD}}\left[H(t)+K_{\mathrm{MSD}} G(t)^{2}+\sigma \hat{K}_{\mathrm{MSD}}\right] .
\end{aligned}
$$

Here $r_{s c}^{2}$ follows from Eq. (23) with $f$ and $f^{s}$ specialized to $f^{c}$ and $f^{s c}$, respectively, and the other amplitudes read

$$
\begin{aligned}
h_{\mathrm{MSD}}= & r_{s c}^{4}\left[\mathcal{F}_{\mathrm{MSD}}^{c}\left(h, f^{s c}\right)+\mathcal{F}_{\mathrm{MSD}}^{c}\left(f^{c}, h^{s}\right)\right], \\
K_{\mathrm{MSD}}= & \frac{r_{s c}^{4}}{h_{\mathrm{MSD}}}\left[\mathcal{F}_{\mathrm{MSD}}^{c}\left(h, h^{s}\right)+\mathcal{F}_{\mathrm{MSD}}^{c}\left(h K, f^{s c}\right)\right. \\
& \left.+\mathcal{F}_{\mathrm{MSD}}^{c}\left(f^{c}, h^{s} K^{s}\right)\right]-\lambda \frac{h_{\mathrm{MSD}}}{r_{s c}^{2}}, \\
\hat{K}_{\mathrm{MSD}}= & \frac{r_{s c}^{4}}{h_{\mathrm{MSD}}}\left[\frac{\partial \mathcal{F}_{\mathrm{MSD}}^{c}\left(f^{c}, f^{s c}\right)}{C \partial \varepsilon}+\mathcal{F}_{\mathrm{MSD}}^{c}\left(h \hat{K}, f^{s c}\right)\right. \\
& \left.+\mathcal{F}_{\mathrm{MSD}}^{c}\left(f^{c}, h^{s} \hat{K}^{s}\right)\right]-\frac{h_{\mathrm{MSD}}}{r_{s c}^{2}},
\end{aligned}
$$

where we exploited that the mode-coupling functional $\mathcal{F}_{\text {MSD }}\left(f^{c}, f^{s c}\right)$ is linear in $f$ and $f^{s}$, and introduced short-hand notation like $h K$ for $(h K)_{p}=h_{p} K_{p}$. 


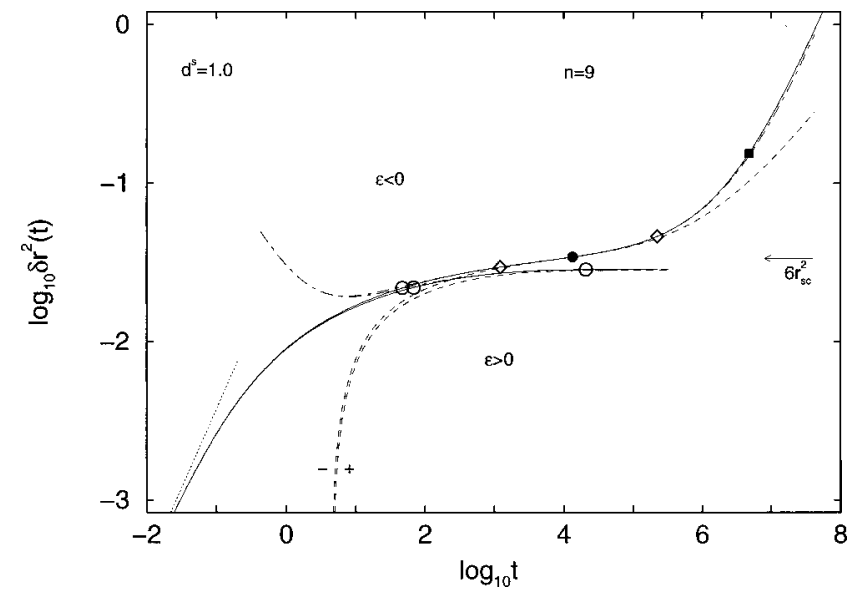

FIG. 7. Mean-square-displacement curves $\delta r^{2}(t)$ (full lines) taken for $n=9$ from Fig. 6 . The dashed and dot-dashed lines, respectively, are the leading and next-to-leading orders of the asymptotic expansion (26) with $r_{s c}^{2}=5.57 \times 10^{-3}, \quad h_{\mathrm{MSD}}$ $=0.0116, \quad K_{\mathrm{MSD}}=-1.23$, and $\hat{K}_{\mathrm{MSD}}=3.33$, calculated from Eqs. (27). The open symbols indicate the range of validity of the first $(\diamond)$ and second $(\bigcirc)$ order formulas as defined at the end of Sec. III C 1 . On the liquid side the second-order range of validity extends beyond the displayed range. On the glass side the first-order range of validity does not exist. The dotted straight line of slope unity for small times indicates the short-time-diffusion asymptote $\left(6 D_{0}^{s} t\right)$.

In Fig. 7 the mean-squared displacement is compared to the asymptotic results in leading and next-to-leading order for a tagged particle in the HSS. The next-to-leading-order result explains the deviations from the leading-order one. The general trend of the deviations is again explained by the short-time corrections to the critical law and the long-time corrections to the von Schweidler law on the liquid side-or to the nonergodicity parameter $6 r_{s c}^{2}$ on the glass side. The range of validity is smaller than for any of the $q$ vectors discussed in connection with Fig. 4, since the absolute value of $K_{\mathrm{MSD}}$ is larger than of all the $K_{i}^{s}(i=0,1,2$, and 3$)$. The next-to-leading-order results are a considerable improvement over the leading ones: On the glass side, the second-order range of validity extends over almost three decades, while the first-order range-as defined at the end of Sec. III C 1does not even exist. On the liquid side the next-to-leading order adds about a decade to the short-time side of the range of validity. It extends it beyond the boundaries of the figure on the long-time side. However, this happens in part by accident because the exponent of the second term $\left(t^{2 b}, 2 b\right.$ $=1.16)$ in the von Schweidler series is close to the exact long-time exponent, given by the diffusion law $\left(6 D^{s} t\right)$.

\section{2. $\alpha$ relaxation}

A description of the $\alpha$ process for the mean-squareddisplacement dynamics can be derived from Eq. (19). Thereby one obtains the superposition principle

$$
\delta r^{2}(t)=\delta \widetilde{r}^{2}\left(t / t_{\sigma}^{\prime}\right)
$$

Here the master function $\delta \widetilde{r}^{2}$ is to be calculated from

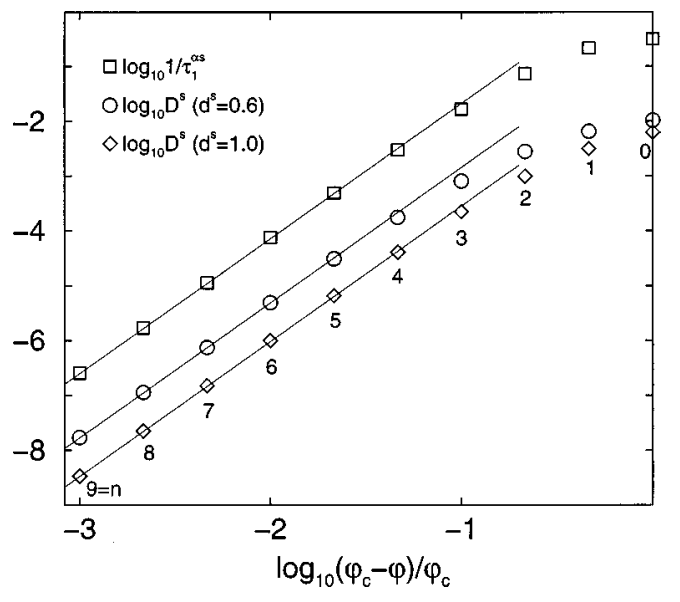

FIG. 8. Self-diffusion coefficients $D^{s}$ for tagged particles of diameters $d^{s}=1.0$ and 0.6 and $\alpha$-relaxation rate $1 / \tau_{1}^{\alpha s}$ vs the reduced packing fraction $\varepsilon=\left(\varphi-\varphi_{c}\right) / \varphi_{c}$. The solid lines are the power-law asymptotes $\left(\Gamma_{A}|\varepsilon|^{\gamma}, \quad \gamma=2.46\right)$. The $\alpha$-scaling time $\tau_{1}^{\alpha s}$ is defined as the time when the tagged-particle density correlator $\Phi_{1}^{s}(t)$, shown in Fig. 3, has relaxed to half of its critical plateau value $f_{1}^{s c}=0.760$.

$$
\int_{0}^{\tilde{t}} m^{(0) c}\left(\tilde{t}-t^{\prime}\right) \delta \tilde{r}^{2}\left(t^{\prime}\right) d t^{\prime}=6 \tilde{t}
$$

with the initial condition $\delta \widetilde{r}^{2}(\tilde{t})=6\left[r_{s c}^{2}+h_{\mathrm{MSD}} \tilde{t}^{b}\right]$ $+\mathcal{O}\left(\widetilde{t}^{2 b}\right)$. The kernel is given by the mode-coupling functional at the critical point $\mathcal{F}_{\text {MSD }}^{c}$ and by the densityfluctuation master functions $m^{(0) c}(\tilde{t})=\mathcal{F}_{\mathrm{MSD}}^{c}\left(\tilde{\Phi}(\tilde{t}), \widetilde{\Phi}^{s}(\tilde{t})\right)$.

The various superposition principles imply coupling of the $\alpha$-relaxation time scales or relaxation rates in the following sense [3]. Let us characterize the long-time decay of the variable $A$ in the liquid by some time $\tau_{A}$. This time diverges upon approaching the glass transition: in the leading asymptotic limit for $\sigma \rightarrow 0-$, one finds $\tau_{A}=C_{A} t_{\sigma}^{\prime}$. All times or rates are proportional to each other and follow a power law, specified by the exponent $\gamma: 1 / \tau_{A}=\Gamma_{A}|\varepsilon|^{\gamma}$ [Eq. (2)]. The constants of proportionality $C_{A}$ or $\Gamma_{A}$ depend on the variable $A$ and on the precise convention for the definition of $\tau_{A}$. The scale coupling or $\alpha$-scale universality is demonstrated in Fig. 8, where the diffusion coefficients of hardsphere particles of diameters $d^{s}=1.0$ and $d^{s}=0.6$, and the $\alpha$-scaling rate $1 / \tau_{1}^{\alpha s}$ of the density fluctuations for wave vector $q_{1}$ are compared to the asymptotic predictions. Although the asymptotic behavior is the same for all the quantities, $D^{s}$ for $d^{s}=0.6$ already deviates visibly from the asymptotic result for $n=4$, while the other quantities start to deviate only for $n=3$. This again underlines the nonuniversality of the deviations. Note that this difference might not be due to asymptotic corrections in the sense discussed in this paper, but could come from the mismatch of structural relaxation, governed by the MCT kernel $m_{q}^{s}(t)$, and the transient dynamics, ruled by the times $\tau_{q}^{s}$ in Eq. (9).

In Fig. 9 the rescaled mean-squared displacement is compared to the $\alpha$ master function $\delta \tilde{r}{ }^{2}(\tilde{t})$ for various packing fractions. The time scale $t_{\sigma}^{\prime}$ is taken from Eq. (2). Note that this figure is a harder test of the asymptotics then just testing 


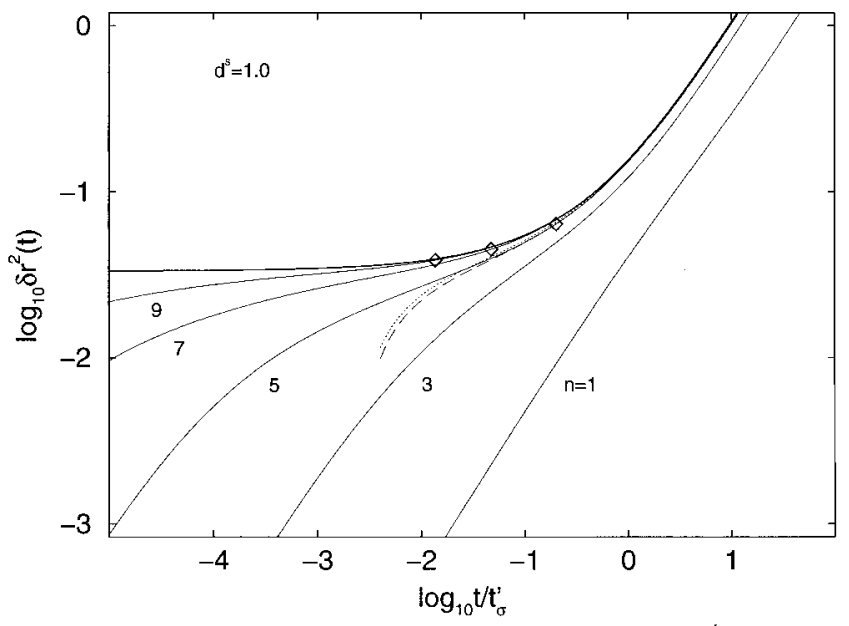

FIG. 9. $\alpha$-scaling plot of the mean-square displacement $\delta r^{2}(t)$, taken from Fig. 6, for reduced packing fractions $\varepsilon=\left(\varphi-\varphi_{c}\right) / \varphi_{c}$ $=-10^{-n / 3}, n=1,3,5,7$, and 9 , vs rescaled time $\tilde{t}=t / t_{\sigma}^{\prime}$. The thick solid line is the $\alpha$ master function $\delta \widetilde{r}^{2}(\tilde{t})$ calculated from Eq. (29). The diamonds mark the early-time bound for the $\alpha$-scaling regime as defined at the end of Sec. III C 1. The dashed line shows the master function corrected according to the analog of Eq. (18). Leaving aside the $\tilde{t}$-independent bracket term, one obtains the dotted curves.

the time-density superposition principle, since it tests the scaling time $t_{\sigma}^{\prime}$ in addition to the shape of the master function.

The leading correction to Eq. (28) is known to be of order $|\sigma|$, while the leading corrections to the factorization property are of relative order $\sqrt{|\sigma|}$ [18], which explains why $\alpha$ scaling works much better than $\beta$ scaling. As we already pointed out in Sec. III C 2, the "quality" of $\alpha$ scaling is observable independent—as can be seen by comparing Fig. 9 to Fig. 5-even though first-order $\beta$ scaling is much worse for $\delta r^{2}(t)$ than for $\Phi_{1}^{s}(t)$. This is so because the dominant correction to the long-time $\beta$ process is the corrections to the von Schweidler law (see Fig. 7), which is absorbed into the short-time expansion of the $\alpha$ master function. The dashed and dotted curves in Fig. 9 show how the deviations from the superposition principle for small rescaled times $\tilde{t}$ are explained by Eq. (18).

\section{GAUSSIAN APPROXIMATION}

The small- $q$ expansion of the density correlator $\Phi_{q}^{s}(t)$, which lead us to the equation of motion for the meansquared displacement, can be viewed as the leading term of the Gaussian approximation [36-38]

$$
\Phi_{q}^{s}(t) \approx e^{-q^{2} \delta r^{2}(t) / 6} .
$$

The Gaussian approximation becomes exact for the shorttime dynamics (either ballistic or-as in our case-diffusive motion) and for the long-time diffusion, and is known to work quite well for simple liquids such as argon in its normal state [38]. So in the following we are primarily interested in the intermediate time window of the structural-relaxation regime.

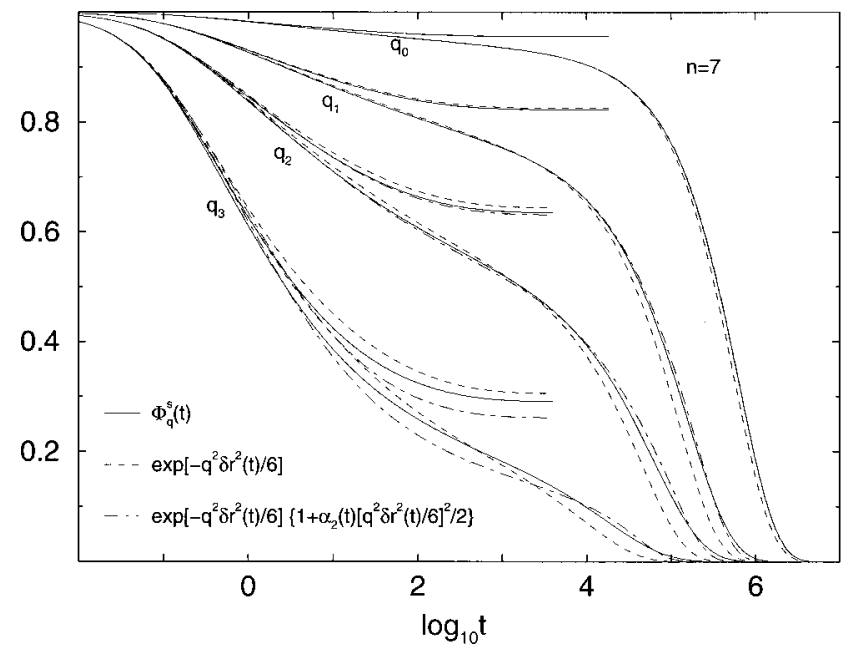

FIG. 10. Test of the Gaussian approximation (30) (dashed lines) and of the cumulant expansion (32) (dot-dashed lines) for the selfintermediate scattering function of the HSS (solid lines) at packing fraction $\varphi=\varphi_{c}\left(1 \pm 10^{-7 / 3}\right)$ for the wave numbers $q_{0}, q_{1}, q_{2}$, and $q_{3}$ introduced in Fig. 1 .

In Fig. 10, some tagged-particle correlators as calculated for $|\varepsilon|=10^{-7 / 3}$ are compared with the Gaussian approximation. The latter describes the behavior of the selfintermediate scattering function of the HSS reasonably for all considered wave numbers. Coming from small wave numbers, at which the Gaussian approximation is asymptotically exact, the deviations start to appear at the end of the $\beta$-relaxation regime, i.e., in the von Schweidler region as exemplified for $q=q_{0}$. Going to still larger wave numbers like $q_{2}$ and $q_{3}$, the Gaussian approximation also deviates at the beginning of the $\beta$-relaxation regime, i.e., in the critical region.

The Gaussian approximation for the critical amplitudes can be found by substituting Eq. (26) into Eq. (30), and expanding the exponential:

$$
\begin{gathered}
f_{q}^{s c} \approx e^{-q^{2} r_{s c}^{2}}, \\
h_{q}^{s} \approx e^{-q^{2} r_{s c}^{2} q^{2} h_{\mathrm{MSD}},} \\
K_{q}^{s} \approx K_{\mathrm{MSD}}+\frac{1}{2} q^{2} h_{\mathrm{MSD}}, \\
\bar{K}_{q}^{s} \approx \bar{K}_{\mathrm{MSD}}+\frac{1}{2 \sqrt{1-\lambda}} q^{2} h_{\mathrm{MSD}} .
\end{gathered}
$$

Here $\bar{K}_{\mathrm{MSD}}$ has been introduced in analogy to Eq. (17). These approximation results are compared to the various amplitudes in Figs. 1 and 2. In both cases we find a good qualitative description for the amplitudes; the Gaussian approximation even describes the change of sign of $K_{q}^{s}$ and $\bar{K}_{q}^{s}$. The Gaussian results for $f^{s c}$ and $h^{s}$ are in better quantitative agreement with the exact results than the results for $K_{q}^{s}$ and $\bar{K}_{q}^{s}$, since the leading small- $q$ corrections to Eqs. (31a) and (31b) are of higher order than those for Eqs. (31c) and (31d). Obviously the results for $d^{s}=1.0$ are better than for the smaller particle with $d^{s}=0.6$. For the large particle they even give good quantitative descriptions for some of the ampli- 
tudes: practically no deviation can be found for the critical Lamb-Mössbauer factor $f^{s c}$ up to $q=15$, and the critical amplitude $h^{s}$ is reproduced up to $q=5$.

These results also explain the deviations for the dynamics in Fig. 10: Since the critical amplitude $h^{s}$ is overestimated by the Gaussian approximation, the dashed curves relax faster in the $\beta$-relaxation region. On the glass side, a larger $h^{s}$ leads to a larger $f^{s}$ via $f^{s}=f^{s c}+h^{s} \sqrt{\sigma /(1-\lambda)}$. In the same way the corresponding figure for $d^{s}=0.6$ can be inferred from Fig. 2.

\section{NON-GAUSSIAN PARAMETER}

\section{A. Equation of motion}

A cumulant expansion can be used systematically to classify the deviations of the density correlator from its Gaussian approximation. The leading contribution to the deviations is proportional to the non-Gaussian parameter (NGP) $\alpha_{2}(t)$. One gets

$$
\Phi_{q}^{s}(t)=e^{-q^{2} \delta r^{2}(t) / 6}\left\{1+\frac{1}{2} \alpha_{2}(t)\left[q^{2} \delta r^{2}(t) / 6\right]^{2}+O\left(q^{6}\right)\right\},
$$

where $\quad \alpha_{2}=\frac{3}{5}\left[\delta r^{4}(t) / \delta r^{2}(t)^{2}\right]-1, \quad$ with $\quad \delta r^{4}(t)=\langle| \vec{r}(t)$ $\left.-\left.\vec{r}(0)\right|^{4}\right\rangle[36-38]$. Since the mean-quartic displacement $\delta r^{4}(t)$ is proportional to the fourth Taylor coefficient in a small- $q$ expansion of $\Phi_{q}^{s}(t)=1-q^{2} \delta r^{2}(t) / 3 !+q^{4} \delta r^{4}(t) / 5$ ! $+\cdots$, one can derive an equation of motion for $\alpha_{2}$ from Eqs. (9) and (19):

$$
\begin{aligned}
{[1+} & \left.\alpha_{2}(t)\right] \delta r^{2}(t)^{2} \\
& +D_{0}^{s} \int_{0}^{t} m^{(0)}\left(t-t^{\prime}\right)\left[1+\alpha_{2}\left(t^{\prime}\right)\right] \delta r^{2}\left(t^{\prime}\right)^{2} d t^{\prime} \\
= & 6 D_{0}^{s} \int_{0}^{t}\left[2+m^{(2)}\left(t-t^{\prime}\right)\right] \delta r^{2}\left(t^{\prime}\right) d t^{\prime} .
\end{aligned}
$$

Here a further mode-coupling functional for the kernel $m^{(2)}(t)=\mathcal{F}_{\mathrm{NGP}}\left(\Phi(t), \Phi^{s}(t)\right)$ is introduced:

$$
\mathcal{F}_{\mathrm{NGP}}\left(f, f^{s}\right)=\frac{1}{10 \pi^{2}} \int n S_{k} c_{k}^{s 2} k^{4} f_{k}\left(\frac{\partial^{2} f_{k}^{s}}{\partial k^{2}}+\frac{2}{3 k} \frac{\partial f_{k}^{s}}{\partial k}\right) d k .
$$

For a numerical calculation, integral (34) is expressed by a Riemann sum analogous to Eq. (21). The derivatives $\partial f_{k}^{s} / \partial k$ and $\partial^{2} f_{k}^{s} / \partial k^{2}$ are approximated through their numerical equivalents, e.g., $\partial f_{k}^{s} / \partial k=\left(f_{k+\Delta}-f_{k-\Delta}\right) /(2 \Delta)$, except for $q=0.2$ and 0.6 , where they are calculated from a small- $q$ extrapolation according to the Gaussian approximation. For the same reasons as discussed above in connection with Eqs. (19), (20), and (21), it is preferable to solve the derived equation for $\alpha_{2}(t)$ directly rather than to deduce the nonGaussian parameter as the small- $q$ limit from the numerical results for $\Phi_{q}^{s}(t)$. We have nevertheless checked that the latter procedure can be followed, provided one chooses a finer wave-vector grid and a proper large- $q$ regularization of integral (10).

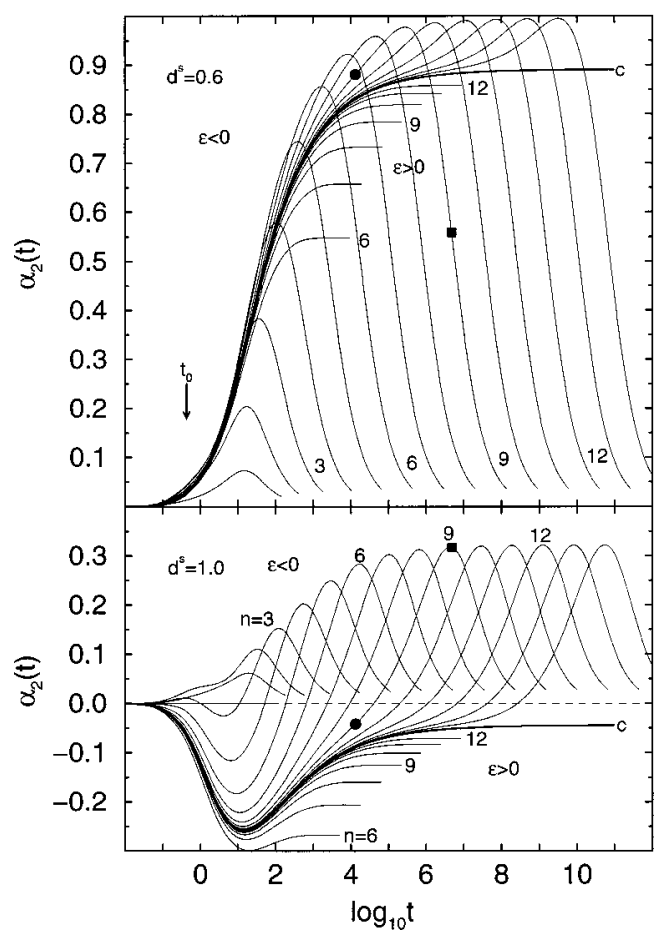

FIG. 11. Non-Gaussian parameter $\alpha_{2}(t)$, obtained from Eq. (33), for tagged particles of diameters $d^{s}=1.0$ and 0.6. The labeling is done as in Fig. 3.

\section{B. Transition scenario}

To begin with, some comments concerning the general properties of $\alpha_{2}(t)$ might be appropriate. Neither $\delta r^{2}(t)$ nor $\alpha_{2}(t)$ are correlation functions and, therefore, the known general properties of positive definite functions like $\Phi_{q}(t)$ and $\Phi_{q}^{s}(t)$ need not be valid for these quantities. With $A(t)$ $=|\vec{r}(t)-\vec{r}(0)|^{2}$ denoting a positive observable, one obtains $\delta r^{2}(t)=\langle A(t)\rangle$, and therefore $\delta r^{2}(t) \geqslant 0$. Since $\Phi_{q}^{s}(t)=1$ $-q^{2} \delta r^{2}(t) / 6+O\left(q^{4}\right)$, for completely monotone density correlators one finds $-(-\partial / \partial t)^{l} \delta r^{2}(t) \geqslant 0$ for $l=1,2, \ldots$. So within the structural relaxation window $t \gg t_{0}$, and for all $t$, for our colloid model defined by Eqs. (1) and (9), the meansquared displacement is an increasing function of time: $(\partial / \partial t) \delta r^{2}(t) \geqslant 0$. The inequality $\left\langle A^{2}\right\rangle \geqslant\langle A\rangle^{2}$ implies $\alpha_{2}(t)$ $\geqslant-2.5$. Hence the non-Gaussian parameter is bounded from below, but it can have either sign. At early times the density correlator exhibits Gaussian behavior, independent of whether Newtonian or Brownian dynamics is considered, and therefore $\alpha_{2}(t \rightarrow 0)=0$. The long-time liquid correlators describe diffusion, which is a Gaussian process, and thus $\alpha_{2}(t \rightarrow \infty)=0$. From Eqs. (33) and (19) one finds for $\sigma<0$ that $\alpha_{2}(t \rightarrow \infty)=O(1 / t)$. Hence in the liquid state $\alpha_{2}(t)$ cannot be a monotonic function of time. Negative $\alpha_{2}(t)$ means that the probability for the particle to move very far is suppressed relative to the one expected for a random-walk process. Similarly, the probability for moving far is enhanced if $\alpha_{2}(t)>0$. In the latter case the cage boundary is more fuzzy than in the former.

Figure 11 exhibits the evolution of the non-Gaussian parameter $\alpha_{2}(t)$ upon crossing the bifurcation point for tagged spheres of diameters $d^{s}=1.0$ and $d^{s}=0.6$ in the HSS. For the glass state the curves exhibit arrest for late times $\alpha_{2}(t \rightarrow \infty)$ 
$=f_{\mathrm{NGP}}=1+\mathcal{F}_{\mathrm{NGP}}\left(f, f^{s}\right)$. For the liquid state the $\alpha$ process manifests itself as a bump of the $\alpha_{2}$-versus- $\log _{10} t$ diagram starting with an increase above the plateau $f_{\mathrm{NGP}}^{c}$, reaching some maximum, and then decreasing to zero. The liquid curves for $n \geqslant 10$, i.e., for $|\varepsilon|<10^{-3}$, exhibit the superposition principle: a change of $\sigma$ causes a shift of the peak parallel to the logarithmic time axis without change of the shape. The first structural relaxation step deals with the approach toward $f_{\mathrm{NGP}}^{c}$ from below for $t \gg t_{0}$. The functions for $|\varepsilon| \leqslant 10^{-4}$ exhibit the two-step-relaxation scenario for $t$ $\gtrsim 10^{4}$.

For dense normal liquids $\alpha_{2}$ is found to be positive, in agreement with molecular dynamics results for liquid argon near its triple point. Our results for $d^{s}=1.0$ and $n=2$ are of about the same magnitude as the value 0.13 found for this Lennard-Jones system [38]. Increasing the density toward the critical one implies an increase of $\alpha_{2}$ by about a factor 3 for both values of $d^{s}$ studied, but for the smaller spheres the non-Gaussian parameter is about three times larger than for the larger ones. One concludes that glassy dynamics in the liquid does not lead to dramatic changes of the magnitude of the non-Gaussian parameter, a finding also supported by Fig. 10.

For long times, and for the smaller sphere for all times, $\alpha_{2}$ is positive. This enhanced probability for the particle to move further is what one would expect as a result of the building of a backflow pattern in the liquid. However, for $d^{s}=1.0$ the negative plateau value $f_{\mathrm{NGP}}^{c}$ implies that for sufficiently large densities a dip to negative values for $\alpha_{2}(t)$ appears. For $d^{s}=0.6$ the plateau is positive and the dip does not exist. The depth of the predicted dip, as opposed to the height of the maximum in the $\alpha$ regime, is not a structural relaxation phenomenon. Rather, it is caused by the cross over from the short-time transient to the first structural relaxation step. The mode-coupling approximations are constructed to describe the long-time behavior due to the cage effect. The theory does not handle short-time collision effects correctly, and therefore the size of the dip predicted for $d^{s}=1.0 \mathrm{might}$ be an artifact due to the insufficiencies of the MCT in a regime, which it was not made for. An analogous reservation applies, of course, to the quantitative details of the crossover regimes displayed in Fig. 3 for $\Phi_{q}^{s}(t)$, and in Fig. 6 for $\delta r^{2}(t)$. The MCT equations do not guarantee the validity of the inequality $\alpha_{2}(t) \geqslant-2.5$. Indeed, for $d^{s}=1.0$ and $\varphi>\varphi_{c}$ +0.01 the inequality is violated, and therefore the corresponding glass curves are not shown in Fig. 11.

The extension of the Gaussian approximation according to Eq. (32) is illustrated by the dash-dotted lines in Fig. 10.
For the wave vectors $q \leqslant q_{2}$, where the Gaussian approximation yields a good description of the correlators, the addition of the leading-cumulant correction proportional to $\alpha_{2}(t)$ improves the fit seriously. However, for larger wave vectors the addition of the $\alpha_{2}$ term does not lead to improvements, as is demonstrated for wave vector $q_{3}$ in Fig. 10. As $\alpha_{2}$ is considerably larger for $d^{s}=0.6$ than for $d^{s}=1.0$, the cumulant expansion (32) already breaks down for $q$ near $q_{0}$.

Figures 3, 6, and 11 have been shown in order to illustrate the theoretical essence of the MCT bifurcation. To avoid misleading conclusions from Fig. 11, it might be adequate to remember the windows accessible by state-of-the-art experimental studies. In molecular-dynamics work $[11,25]$ a variation of the diffusivity or other structural relaxation scales could be detected over a window of about four decades. This corresponds to the curves $n \leq 7$ in our figures. The dynamical window for structural relaxation explored by van Megen and Underwood [9] by photon-correlation spectroscopy is also about four decades wide. The size of the dynamical window accessible by the neutron-spin-echo instrument [5] is smaller than three decades. To focus on results for $\alpha_{2}$ which might be relevant for the interpretation of experiments with the techniques available today, one should ignore the results for $n \geq 8$ in Fig. 11. This restricted set of results exhibits neither the superposition principle nor the two-step relaxation scenario. The leading-order asymptotic results for the MCT bifurcation dynamics do not describe the MCT results shown in Fig. 11 for $n \lesssim 8$, i.e., for $|\varepsilon|>0.001$, not even qualitatively. But in the following it shall be shown that the results can be understood in terms of the next-to-leading asymptotic laws.

\section{Asymptotic laws}

\section{1. $\beta$ relaxation}

In order to work out the dynamics in the $\beta$-relaxation window, we start from the Laplace transform of Eq. (33):

$$
\begin{aligned}
\mathcal{L}\left[\left(1+\alpha_{2}(t)\right) \delta r^{2}(t)^{2}\right](s) \\
\quad=s \delta r^{2}(s)^{2}\left\{2+s \mathcal{L}\left[\mathcal{F}_{\mathrm{NGP}}\left(\Phi(t), \Phi^{s}(t)\right)\right](s)\right\},
\end{aligned}
$$

where the Laplace transform of Eq. (19) has been used. Inserting the asymptotic expansions (6), (14), and (26) for $\Phi_{q}(t), \Phi_{q}^{s}(t)$, and $\delta r^{2}(t)$, respectively, one obtains

$$
\begin{aligned}
\alpha_{2}(t)= & f_{\mathrm{NGP}}+h_{\mathrm{NGP}} G(t) \\
& +h_{\mathrm{NGP}}\left[H(t)+K_{\mathrm{NGP}} G(t)^{2}+\sigma \hat{K}_{\mathrm{NGP}}\right] .
\end{aligned}
$$

Here the amplitudes are given by

$$
\begin{gathered}
h_{\mathrm{NGP}}=\mathcal{F}_{\mathrm{NGP}}^{c}\left(h, f^{s c}\right)+\mathcal{F}_{\mathrm{NGP}}^{c}\left(f^{c}, h^{s}\right), \\
K_{\mathrm{NGP}}=\left[\mathcal{F}_{\mathrm{NGP}}^{c}\left(h, h^{s}\right)+\mathcal{F}_{\mathrm{NGP}}^{c}\left(h K, f^{s c}\right)+\mathcal{F}_{\mathrm{NGP}}^{c}\left(f^{c}, h^{s} K^{s}\right)\right] / h_{\mathrm{NGP}}+\frac{h_{\mathrm{MSD}}}{r_{s c}^{4}}(1-\lambda)\left[2 r_{s c}^{2} h_{\mathrm{NGP}}-h_{\mathrm{MSD}}\left(1+f_{\mathrm{NGP}}\right)\right] / h_{\mathrm{NGP}}, \\
\hat{K}_{\mathrm{NGP}}=\left[\frac{\partial \mathcal{F}_{\mathrm{NGP}}^{c}\left(f^{c}, f^{s c}\right)}{C \partial \varepsilon}+\mathcal{F}_{\mathrm{NGP}}^{c}\left(h \hat{K}, f^{s c}\right)+\mathcal{F}_{\mathrm{NGP}}^{c}\left(f^{c}, h^{s} \hat{K}^{s}\right)\right] / h_{\mathrm{NGP}}+\frac{h_{\mathrm{MSD}}}{r_{s c}^{4}}\left[h_{\mathrm{MSD}}\left(1+f_{\mathrm{NGP}}\right)-2 r_{s c}^{2} h_{\mathrm{NGP}}\right] / h_{\mathrm{NGP}} .
\end{gathered}
$$




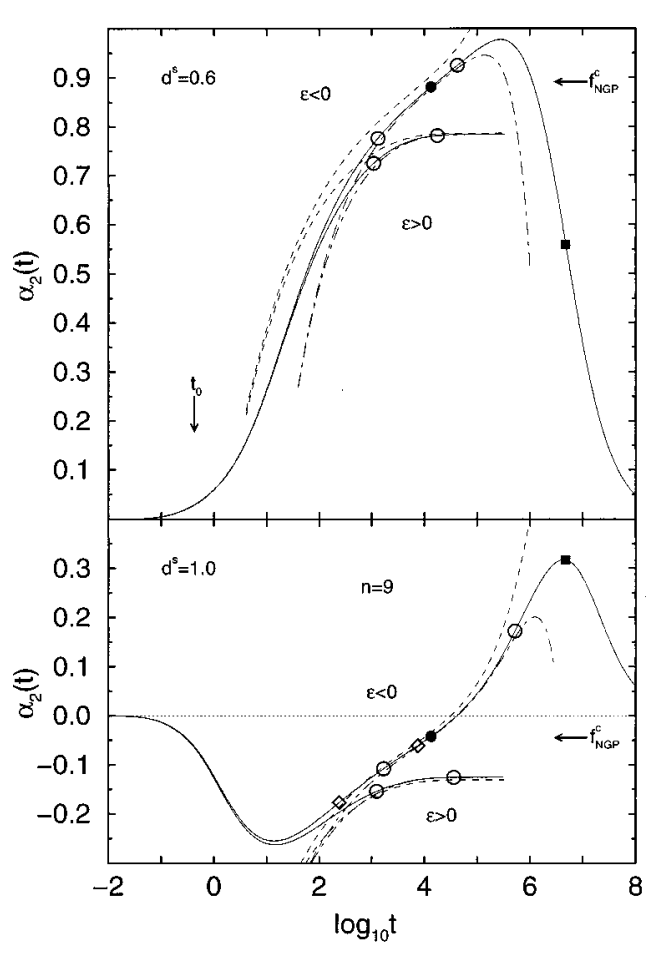

FIG. 12. $\beta$-relaxation behavior of the non-Gaussian parameter $\alpha_{2}(t)$ (full lines) taken for $n=9$ from Fig. 11 . The dashed and dot-dashed lines, respectively, are the leading- and nextto-leading-order asymptotic expansion (36). The critical amplitudes $\quad\left(d^{s}=1.0: \quad f_{\mathrm{NGP}}^{c}=-0.0445, \quad h_{\mathrm{NGP}}=-1.12, \quad K_{\mathrm{NGP}}\right.$ $=0.543, \quad$ and $\quad \hat{K}_{\mathrm{NGP}}=-2.98 ; \quad d^{s}=0.6: \quad f_{\mathrm{NGP}}^{c}=0.891, \quad h_{\mathrm{NGP}}$ $=-1.37, K_{\mathrm{NGP}}=3.61$, and $\left.\hat{K}_{\mathrm{NGP}}=-9.85\right)$ were calculated from Eqs. (37). The symbols indicate the range of validity of the leading $(\diamond)$ and the next-to-leading $(O)$ order as defined at the end of Sec. III C 1-the first-order range of validity indicated in the lower panel is due to an "accidental" intersection of curves.

The analytic results are compared with the numerical solutions for $n=9$ in Fig. 12. Because of the large negative value of $h_{\mathrm{NGP}}$, the leading order qualitatively accounts for the steep rise of $\alpha_{2}$ in the $\beta$-relaxation region. This is a major reason for the absence of a plateau even though the reduced packing fraction $|\varepsilon|=0.001$ is so small. The large size of $h_{\mathrm{NGP}}$ also explains why the long-time limit $f_{\mathrm{NGP}}$ in the glass is much more suppressed below the plateau value $f_{\mathrm{NGP}}^{c}$, than shown in Fig. 7 for $\delta r^{2}(t)$. There are two further peculiarities hidden in Eqs. (37), which render the discussion of Fig. 12 different from those considered so far for other examples. The $\alpha$ process for the density correlators and for the meansquared displacement deals with monotonic functions. In those cases the leading corrections to the von Schweidler law influence the details but not the general trend of the functions. The positive slope of $\alpha_{2}(t)$ in the leading-order $\beta$-relaxation regime, however, has to change to a negative one in the late $\alpha$-relaxation regime since the $\alpha_{2}(t)$-versus- $t$ curve eventually has to decrease to zero. Therefore the $\alpha_{2}(t)$-versus- $\log _{10} t$ diagram exhibits a bump. The next-toleading asymptotic formula (36), as opposed to the leadingorder one, can reproduce at least qualitatively this new feature of the diagram as shown by the dot-dashed lines in Fig. 12. The second difference concerns the remarkable parallel shift of the solution relative to the leading asymptote occur- ring for times near $t_{\sigma}^{-}$where the liquid $\beta$ correlator exhibits a zero, $G\left(t_{\sigma}^{-}\right)=0\left(t_{\sigma}^{-}=0.704 t_{\sigma}\right.$ for the HSS). The correction is given by $h_{\mathrm{NGP}}\left[H\left(t_{\sigma}^{-}\right)+\sigma \hat{K}_{\mathrm{NGP}}\right]$. For the previously discussed examples the two terms in the brackets partly canceled each other, while for $\alpha_{2}(t)$ they add up. As a result this shift is about an order of magnitude larger than in the examples studied in Figs. 4 and 7, or in the examples considered in Ref. [18]. This shift is responsible for the fact that for $n=9$ the leading-order asymptotic result for $\alpha_{2}(t)\left(d^{s}=1\right)$ (dashed lines in the lower panel of Fig. 12) has a smaller range of validity than the corresponding result for the mean-square displacement (Fig. 7) even though $\left|K_{\mathrm{NGP}}\right|$ $<\left|K_{\mathrm{MSD}}\right|$. Of course, this does not contradict the idea that the amplitude $K$ asymptotically determines the range of validity of the leading-order result, because, asymptotically close to the critical point, the shift becomes irrelevant. Thus the leading-order range of validity for $\alpha_{2}(t)\left(d^{s}=1\right)$ will finally overtake that for $\delta r^{2}(t)$ as $\sigma \rightarrow 0$.

One can substitute expansions (26) and (36) into Eq. (32) in order to obtain the amplitudes in Eq. (14). The result extends the Gaussian approximation (31) for these quantities, so that the first cumulant is taken care of. The result improves the Gaussian approximation for $q \lessgtr q_{0}$. However, for large wave vectors the extended approximation is worse than the Gaussian one, which is shown in Figs. 1 and 2 by the full lines. Therefore the described extension is not worthwhile.

\section{2. $\alpha$ relaxation}

To explore the asymptotic behavior in the $\alpha$-relaxation region, the non-Gaussian parameter is plotted versus rescaled time $\tilde{t}=t / t_{\sigma}^{\prime}$ in Fig. 13. Comparing the results for $d^{s}=1.0$ with the corresponding ones for the mean-squared displacement in Fig. 9, one notices that one has to choose a considerably smaller separation $|\sigma|$ for $\alpha_{2}(t)$ than for $\delta r^{2}(t)$ to find comparable agreement with the $\alpha$-relaxation scaling law. For $n=5$ and $\tilde{t}=1, \delta r^{2}(t)$ agrees with the superposition-principle asymptote within the accuracy of the drawing in Fig. 9, while the corresponding result for $\alpha_{2}$ deviates seriously from the asymptote. The mean-squared displacement is dominated by the diffusion limit, i.e., Gaussian behavior for $\tilde{t}>20$. Such contribution is absent in $\alpha_{2}$, and therefore the $n=5$ curve in Fig. 13 magnifies the small deviations from Gaussian dynamics, which for the $n=5$ curve in Fig. 9 start to be visible only for $\tilde{t} \lesssim 0.3$.

Figures 13 and 9 are seemingly in contradiction to the statement that $\alpha$ scaling should work equally well for different quantities (see Sec. III C 2). However, as mentioned above, the non-Gaussian parameter for the packing fractions shown is special in the sense that the corrections for small $\tilde{t}$ contain an important shift term, which stems from the term in brackets in the short-time expansion of the $\alpha$-scaling correction (18). In Fig. 13 this finding is illustrated for the curves with the label $n=9$. For even smaller $|\sigma|$ this shift becomes irrelevant, thus resolving the paradox.

\section{CONCLUSIONS}

In this paper the MCT results for the structural relaxation of the mean-squared displacement $\delta r^{2}(t)$ and non-Gaussian 


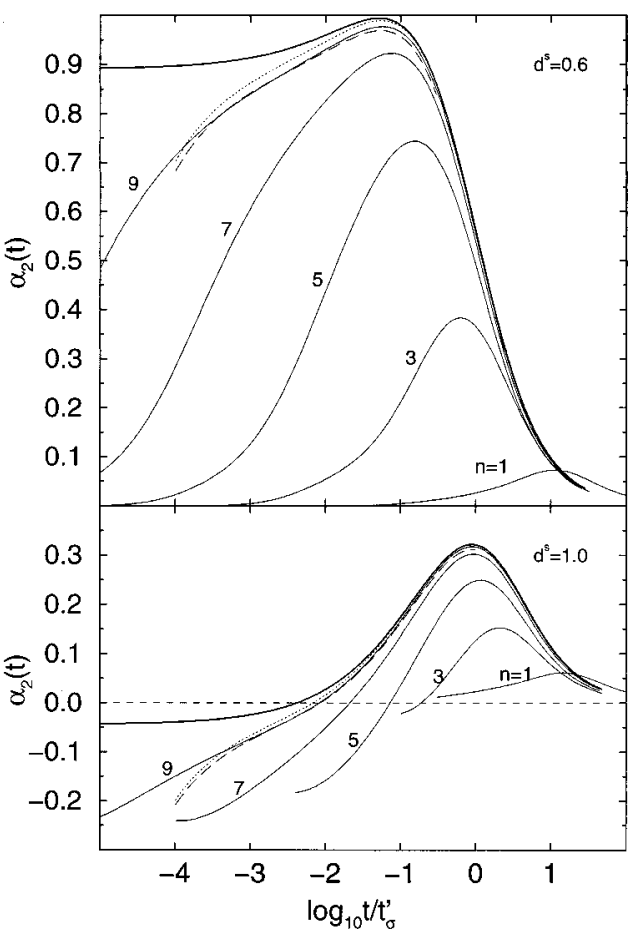

FIG. 13. $\alpha$-scaling plot of the non-Gaussian parameter $\alpha_{2}(t)$, taken from Fig. 11, for reduced packing fractions $\varepsilon=\left(\varphi-\varphi_{c}\right) / \varphi_{c}$ $= \pm 10^{-n / 3}, n=1,3,5,7$, and 9 , vs rescaled time $\tilde{t}=t / t_{\sigma}^{\prime}$. The thick solid line is the $\alpha$ master function $\tilde{\alpha}_{2}(\tilde{t})$. The dashed line shows the master function corrected according to the analog of Eq. (18). Leaving aside the $\tilde{t}$-independent bracket term, one obtains the dotted curves.

parameter $\alpha_{2}(t)$ were discussed for the hard-sphere system. More generally, we studied the generating function of these quantities, viz. the incoherent intermediate scattering function or tagged-particle correlator $\Phi_{q}^{s}(t)$. The work is motivated by the distinguished role of these quantities for the interpretation of spectroscopic data and molecular-dynamics simulations of simple glass forming liquids. The essence of the MCT bifurcation scenario for the evolution of structural relaxation is contained in the leading asymptotic laws which deal with the two scaling laws reviewed in Sec. II. Most of the tests of MCT published so far focused on an assessment of these universal results. The outcome of this paper demonstrates, in a drastic manner, that the range of validity of the mentioned asymptotic laws is not universal, but rather depends on the quantity considered. This range of validity can be determined by working out the laws for the leading corrections. Thereby formulas for a refined data analysis are obtained. There are quantities such as $\alpha_{2}(t)$, which are not described at all within the presently accessible dynamical window by the leading asymptotic result. In this case the laws, including the leading-order corrections, are necessary for a qualitative understanding.

From Fig. 4 one infers that it is relatively easy to extract von Schweidler's law for the relaxation at the structurefactor peak position $q_{1}$, while it is more difficult to identify this leading asymptotic law [Eq. (4)] for the larger wave vector $q_{3}$. The behavior of $\Phi_{q}^{s}(t)$ for the intermediate wavevector range $q_{0}-q_{3}$ is quite similar to what was studied for the coherent intermediate scattering function in Ref. [18].
The results, in particular those for the spectra, can therefore be inferred from that earlier work, using the amplitudes from Figs. 1 and 2. Inclusion of the calculated correction terms often extends the range of validity of the asymptotic formulas seriously. Therefore it is advisable to fit the initial part of the $\alpha$ process by the von Schweidler expansion including the $\tilde{t}^{2 b}$ term, as was done recently by Sciortino et al. [25] in their analysis of simulation data for supercooled water. These authors noticed, in particular, that the leading correction term to Eq. (4) was smallest near $q_{1}$. This is in qualitative agreement with Fig. 4, even though there is no a priori reason to expect our hard-sphere-system results to be relevant for an explanation of the details of water.

Figure 7 implies that the $\delta r^{2}(t)$-versus- $\log _{10} t$ diagram exhibits only a small window for von Schweidler's law, and that the window for the analytic description is extended significantly by inclusion of the $\widetilde{t}^{2 b}$ correction term. It is also predicted that the critical decay law cannot be identified for reduced packing fractions $|\varepsilon| \geqslant 0.001$. These findings are similar to what was reported in Ref. [11] for the simulation results for a binary Lennard-Jones mixture.

In agreement with photon-correlation-spectroscopy results for a hard-sphere colloid [22], one infers from Fig. 10, and from the lower panel of Fig. 11, that the Gaussian approximation works very well for a hard-sphere system. This finding is not in conflict with the observation that $\delta r^{2}(t)$ does not exhibit a critical decay law, while $\Phi_{q}^{s}(t)=\exp$ $\left[-q^{2} \delta r^{2}(t) / 6\right]$ does show this leading asymptotic result [Eq. (3)] for $q \sim q_{3}$ (see Fig. 4). The strong $t^{-2 a}$ corrections to $\delta r^{2}(t) / 6 \sim r_{s c}^{2}-h_{\mathrm{MSD}}\left(t_{0} / t\right)^{a}$ nearly cancel those coming from the expansion of $\exp \left[-q^{2} \delta r^{2}(t) / 6\right]$; the relevant correction amplitude $K_{\mathrm{MSD}}$ for $\delta r^{2}(t)$ is therefore larger than that for $\Phi_{3}^{s}(t)$.

The test of MCT against molecular-dynamics-simulation results by Kob and Andersen [11,39,40] gives strong support for the theory. This can be appreciated even more if one considers the comparisons of first-principles MCT calculations of the critical form factors, critical amplitudes, and exponent parameter with the data [41]. The authors also confirmed the power-law singularity for the $\alpha$-relaxation scales $\tau \propto\left|T-T_{c}\right|^{-\gamma}$ with the predicted connection between the exponent $\gamma$ and the von Schweidler exponent $b$ [Eq. (2)]. However, they obtained the result $D \propto\left|T-T_{c}\right|^{\gamma^{\prime}}$ for the diffusivity, with $\gamma^{\prime}<\gamma$, in contradiction to the $\alpha$-scale universality. This observation underlines in particular that the predicted $\alpha$-scale coupling is not a triviality. Figure 8 demonstrates that the corrections to the leading asymptotic law for the diffusivity of a smaller sphere are larger than the ones for the $\alpha$-relaxation scale for a representative intermediate wave vector $q_{1}$. But our finding for the corrections to the hardsphere-system asymptotics is too small to explain the reported results for the cited mixture. The MCT prediction for the universal $\alpha$ scales for the HSS, $\tau_{\alpha} \propto\left[\left|\varphi-\varphi_{c}\right| / \varphi_{c}\right]^{-2.6}$ $\propto 1 / D$, was confirmed in a recent analysis by van Megen et al. [22] of the diffusivity $D$ and the tagged-particlecorrelator $\alpha$ scale $\tau_{\alpha}$ for a hard-sphere colloid.

The results for $\alpha_{2}(t)$ in the upper panel of Fig. 11 are unusual if compared to the corresponding ones for representative density correlators $\Phi_{q}(t)$ or $\Phi_{q}^{s}(t)$. For the curves with $n \lesssim 8$, dealing with the presently in experiments or 
simulations accessible parameters and windows, the two MCT scaling laws [Eqs. (5) and (8)] cannot be identified. Neither is it possible to recognize the two time fractals [Eqs. (3) and (4)]. These findings can be understood by analytical formulas only, if the next-to-leading asymptotic results are appreciated, as shown in Fig. 12. However, these observations are not so surprising for the following reasons. The $\alpha$ process for the correlator $\Phi_{q}(t)$ deals with the monotonic decay from the plateau $f_{q}^{c}$ to zero. This phenomenon was already described within Maxwell's viscoelastic theory, dealing with exponential decay and Gaussian density fluctuations. Glassy dynamics deals with deviations from this phenomenological description. However, for the time range where $\Phi_{q}^{s}(t)<f_{q}^{s c} / 2$, which deals with a major part of the $\Phi_{q}^{s}(t)$-versus- $\log _{10} t$ graph, the phenomenological picture remains essentially valid. In this part the superposition principle already holds for $n \geqslant 5$, as can be inferred from the $\alpha$-scaling analysis in Fig. 5 or from Ref. [18]. Also, for $\alpha_{2}(t)$ the superposition principle works for the mentioned large times, as shown in Fig. 13. But since there Gaussian dynamics is nearly valid, $\alpha_{2}(t)$ is small, and the corresponding part of the $\alpha_{2}$-versus- $\log _{10} t$ diagram merely deals with a not so interesting feature of the figure. The diagram is dominated by the maximum, and this is located near the end of the window of von Schweidler's law. Here the corrections are large compared to the leading terms, and the maximum is only poorly approximated for $n \leq 5$. But Fig. 5 shows that for $\Phi_{q}^{s}(t)$ the $n=5$ results do not follow the $\alpha$-scaling master curve for $t / t_{\sigma}^{\prime}<0.2$. This too can be inferred from the corresponding scaling plot shown as Fig. 17 in Ref. [18] or from Fig. 9 for $\delta r^{2}(t)$. In the latter case the deviations from scaling are judged relative to the underlying elementary background $f_{q}^{c}$ and $6 r_{s c}^{2}$, respectively, and therefore they do not appear as qualitative effects. The non-Gaussian parameter magnifies a small effect, and therefore its approximation by asymptotic formulas is judged differently than for the other functions. Indeed, for the HSS the predicted size $\alpha_{2}(t)$ $<0.3$ is so small that the effect disappears in the data noise of the measurement on colloids [22]. Let us emphasize that the value $f_{\mathrm{NGP}}$ of the plateau was of importance for the preceding discussion. Therefore, it would be of great interest for an assessment of our results to measure $f_{\mathrm{NGP}}$ in the glass state.

The upper panel of Fig. 11 looks similar to what Kob and Andersen reported for their results on a binary mixture [11]. In particular, we also find $\alpha_{2}$ to be larger for smaller particles; the maximum increases from 1.0 to 1.2 if $d^{s}$ is decreased from 0.6 to 0.5 . The clustering of the $\alpha_{2^{-}}$ versus- $\log _{10} t$ graphs on the critical curve, labeled $c$ in Fig. 11, and referred to as a scaling law in Ref. [11], becomes better for $d^{s}=0.5$ than for the result shown for $d^{s}=0.6$. Let us emphasize that the results found are not universal features of the MCT bifurcation dynamics. The apparent scaling is disturbed if $d^{s}$ increases above 0.6 , and it is predicted to be absent for $d^{s}=1.0$ as is shown in the lower panel of Fig. 11.

It was shown by Kob et al. [42] that the $\alpha_{2}$ peak is produced by clusters of particles which move faster than the ones in their neighborhood. Only as few as $5 \%$ of all particles are involved in the formation of these clusters. It would be very surprising if the MCT, which works with averaged quantities like $\Phi_{q}(t)$ and $\Phi_{q}^{s}(t)$, could reproduce such subtlety of the microscopic dynamics. This would hold even more so if speculations on a relation of the cited cluster dynamics to polymer dynamics [43] could be substantiated. On the other hand, the features of the fast clusters identified so far are not in obvious contradiction to the idea that they are representative configurations building up backflow patterns. Moreover, the MCT equations for structural relaxation were originally proposed and tested against experiments for a treatment of backflow phenomena [44]. Obviously it would be helpful to carry out a first-principles MCT calculation of $\alpha_{2}(t)$ for the cited binary mixture along the same lines as done in this paper for the hard-sphere system. Thereby one could clarify whether or not the qualitative agreement between the hard-sphere-system results for $d^{s}$ about 0.6 with the simulation results in Ref. [11] is just an accident.

\section{ACKNOWLEDGMENTS}

We cordially thank Herman Cummins, Walter Kob, and Bill van Megen for stimulating discussions and helpful comments on our manuscript. Our work was supported by Verbund project No. BMBF 03G04TUM and DFG Grant No. Fu309/2-1/2.
[1] E. Leutheusser, Phys. Rev. A 29, 2765 (1984).

[2] U. Bengtzelius, W. Götze, and A. Sjölander, J. Phys. C 17, 5915 (1984).

[3] W. Götze, in Liquids, Freezing and Glass Transition, edited by J.-P. Hansen, D. Levesque, and J. Zinn-Justin (North-Holland, Amsterdam, 1991), p. 287.

[4] W. Götze and L. Sjögren, Rep. Prog. Phys. 55, 241 (1992).

[5] F. Mezei, Ber. Bunsenges. Phys. Chem. 95, 1118 (1991).

[6] G. Li, W. M. Du, X. K. Chen, H. Z. Cummins, and N. J. Tao, Phys. Rev. A 45, 3867 (1992).

[7] E. Bartsch, M. Antonietti, W. Schupp, and H. Sillescu, J. Chem. Phys. 97, 3950 (1992).

[8] W. van Megen and S. M. Underwood, Phys. Rev. Lett. 70, 2766 (1993).
[9] W. van Megen and S. M. Underwood, Phys. Rev. E 49, 4206 (1994).

[10] J. Baschnagel, Phys. Rev. B 49, 135 (1994).

[11] W. Kob and H. C. Andersen, Phys. Rev. E 51, 4626 (1995).

[12] Y. Yang, L. J. Muller, and K. A. Nelson, in Disordered Materials and Interfaces, edited by H. E. Stanley, H. Z. Cummins, D. J. Dorian, and D. L. Johnson, MRS Symposia Proceedings No. 407 (Materials Research Society, Pittsburgh, 1996), p. 145.

[13] J. Ma, D. V. Bout, and M. Berg, Phys. Rev. E 54, 2786 (1996).

[14] P. Gallo, F. Sciortino, P. Tartaglia, and S.-H. Chen, Phys. Rev. Lett. 76, 2730 (1996).

[15] P. Lunkenheimer, A. Pimenov, and A. Loidl, Phys. Rev. Lett. 78, 2995 (1997). 
[16] H. Z. Cummins, G. Li, W. Du, Y. H. Hwang, and G. Q. Shen, Prog. Theor. Phys. 126, 21 (1997).

[17] A. Tölle, H. Schober, J. Wuttke, and F. Fujara, Phys. Rev. E 56, 809 (1997).

[18] T. Franosch, M. Fuchs, W. Götze, M. R. Mayr, and A. P. Singh, Phys. Rev. E 55, 7153 (1997).

[19] R. Schilling and T. Scheidsteger, Phys. Rev. E 56, 2932 (1997).

[20] T. Franosch, M. Fuchs, W. Götze, M. R. Mayr, and A. P. Singh, Phys. Rev. E 56, 5659 (1997).

[21] W. Petry, E. Bartsch, F. Fujara, M. Kiebel, H. Sillescu, and B. Farago, Z. Phys. B 83, 175 (1991).

[22] W. van Megen, T. Mortensen, J. Müller, and S. Williams, Phys. Rev. E (to be published).

[23] R. Zorn, Phys. Rev. B 55, 6249 (1997).

[24] T. Kanaya, I. Tsukushi, and K. Kaji, Prog. Theor. Phys. Suppl. 126, 133 (1997).

[25] F. Sciortino, P. Gallo, P. Tartaglia, and S.-H. Chen, Phys. Rev. E 54, 6331 (1996).

[26] M. Fuchs, Transp. Theory Stat. Phys. 24, 855 (1995).

[27] W. van Megen and P. N. Pusey, Phys. Rev. A 43, 5429 (1991).

[28] W. van Megen, S. M. Underwood, and P. N. Pusey, Phys. Rev. Lett. 67, 1586 (1991).

[29] W. van Megen and S. M. Underwood, Phys. Rev. E 47, 248 (1993).
[30] W. van Megen and S. M. Underwood, Phys. Rev. Lett. 72, 1773 (1994).

[31] W. van Megen, Transp. Theory Stat. Phys. 24, 1017 (1995).

[32] J. L. Lebowitz, Phys. Rev. 133, A895 (1963).

[33] W. Götze and L. Sjögren, J. Math. Anal. Appl. 195, 230 (1995).

[34] T. Franosch and W. Götze, J. Phys.: Condens. Matter 6, 4807 (1994).

[35] T. Franosch, W. Götze, M. R. Mayr, and A. P. Singh, J. NonCryst. Solids (to be published).

[36] J.-P. Hansen and I. R. McDonald, Theory of Simple Liquids, 2nd ed. (Academic, London, 1986).

[37] A. Rahman, K. S. Singwi, and A. Sjölander, Phys. Rev. 126, 986 (1962).

[38] A. Rahman, Phys. Rev. 136, A405 (1964).

[39] W. Kob and H. C. Andersen, Phys. Rev. E 52, 4134 (1995).

[40] W. Kob and H. Andersen, Transp. Theory Stat. Phys. 24, 1179 (1995).

[41] M. Nauroth and W. Kob, Phys. Rev. E 55, 657 (1997).

[42] W. Kob, C. Donati, S. J. Plimpton, P. H. Poole, and S. C. Glotzer, Phys. Rev. Lett. 79, 2827 (1997).

[43] C. Donati, J. F. Douglas, W. Kob, S. J. Plimpton, P. H. Poole, and S. C. Glotzer, Phys. Rev. Lett. 80, 2338 (1998).

[44] W. Götze and M. Lücke, Phys. Rev. B 13, 3825 (1976). 